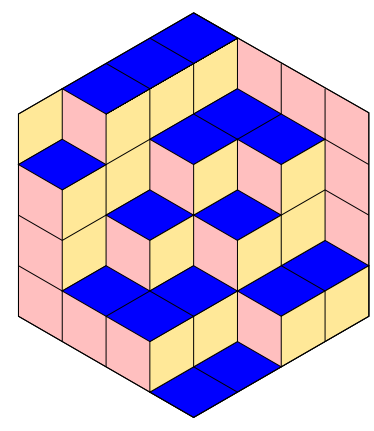

ALGEBRAIC COMBINATORICS

Jia Huang \& Brendon Rhoades

Ordered set partitions and the 0 -Hecke algebra

Volume 1, issue 1 (2018), p. 47-80.

<http://alco.centre-mersenne.org/item/ALCO_2018__1_1_47_0>

(c) The journal and the authors, 2018.

Some rights reserved.

\begin{tabular}{|l|l}
\hline (c) BY This article is licensed under the \\
\hline
\end{tabular}

Creative Commons ATtribution 4.0 InTERnational License.

http://creativecommons.org/licenses/by/4.0/

Access to articles published by the journal Algebraic Combinatorics on the website http://alco.centre-mersenne.org/ implies agreement with the Terms of Use (http://alco.centre-mersenne.org/legal/).

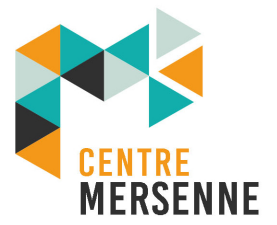

Algebraic Combinatorics is member of the Centre Mersenne for Open Scientific Publishing www.centre-mersenne.org 


\title{
Ordered set partitions and the 0-Hecke algebra
}

\author{
Jia Huang \& Brendon Rhoades
}

\begin{abstract}
Let the symmetric group $\mathfrak{S}_{n}$ act on the polynomial ring $\mathbb{Q}\left[\mathbf{x}_{n}\right]=\mathbb{Q}\left[x_{1}, \ldots, x_{n}\right]$ by variable permutation. The coinvariant algebra is the graded $\mathfrak{S}_{n}$-module $R_{n}:=\mathbb{Q}\left[\mathbf{x}_{n}\right] / I_{n}$, where $I_{n}$ is the ideal in $\mathbb{Q}\left[\mathbf{x}_{n}\right]$ generated by invariant polynomials with vanishing constant term. Haglund, Rhoades, and Shimozono introduced a new quotient $R_{n, k}$ of the polynomial ring $\mathbb{Q}\left[\mathbf{x}_{n}\right]$ depending on two positive integers $k \leqslant n$ which reduces to the classical coinvariant algebra of the symmetric group $\mathfrak{S}_{n}$ when $k=n$. The quotient $R_{n, k}$ carries the structure of a graded $\mathfrak{S}_{n}$-module; Haglund et. al. determine its graded isomorphism type and relate it to the Delta Conjecture in the theory of Macdonald polynomials. We introduce and study a related quotient $S_{n, k}$ of $\mathbb{F}\left[\mathbf{x}_{n}\right]$ which carries a graded action of the 0 -Hecke algebra $H_{n}(0)$, where $\mathbb{F}$ is an arbitrary field. We prove 0-Hecke analogs of the results of Haglund, Rhoades, and Shimozono. In the classical case $k=n$, we recover earlier results of Huang concerning the 0-Hecke action on the coinvariant algebra.
\end{abstract}

\section{INTRODUCTION}

The purpose of this paper is to define and study a 0-Hecke analog of a recently defined graded module for the symmetric group [16]. Our construction has connections with the combinatorics of ordered set partitions and the Delta Conjecture [15] in the theory of Macdonald polynomials.

The symmetric group $\mathfrak{S}_{n}$ acts on the polynomial $\operatorname{ring} \mathbb{Q}\left[\mathbf{x}_{n}\right]:=\mathbb{Q}\left[x_{1}, \ldots, x_{n}\right]$ by variable permutation. The corresponding invariant subring $\mathbb{Q}\left[\mathbf{x}_{n}\right]^{\mathfrak{S}_{n}}$ consists of all $f \in \mathbb{Q}\left[\mathbf{x}_{n}\right]$ with $w(f)=f$ for all $w \in \mathfrak{S}_{n}$, and is generated by the elementary symmetric functions $e_{1}\left(\mathbf{x}_{n}\right), \ldots, e_{n}\left(\mathbf{x}_{n}\right)$, where

$$
e_{d}\left(\mathbf{x}_{n}\right)=e_{d}\left(x_{1}, \ldots, x_{n}\right):=\sum_{1 \leqslant i_{1}<\cdots<i_{d} \leqslant n} x_{i_{1}} \cdots x_{i_{d}} .
$$

The invariant ideal $I_{n} \subseteq \mathbb{Q}\left[\mathbf{x}_{n}\right]$ is the ideal generated by those invariants $\mathbb{Q}\left[\mathbf{x}_{n}\right]_{+}^{\mathfrak{S}_{n}}$ with vanishing constant term:

$$
I_{n}:=\left\langle\mathbb{Q}\left[\mathbf{x}_{n}\right]_{+}^{\mathfrak{S}_{n}}\right\rangle=\left\langle e_{1}\left(\mathbf{x}_{n}\right), \ldots, e_{n}\left(\mathbf{x}_{n}\right)\right\rangle .
$$

The coinvariant algebra $R_{n}:=\mathbb{Q}\left[\mathbf{x}_{n}\right] / I_{n}$ is the corresponding quotient ring.

Manuscript received 9th August 2017, revised 7th December 2017, accepted 11th December 2017. KEYWORDS. Hecke algebra, set partition, coinvariant algebra.

ACKNowledGements. B. Rhoades was partially supported by NSF Grant DMS-1500838. 
The coinvariant algebra $R_{n}$ inherits a graded action of $\mathfrak{S}_{n}$ from $\mathbb{Q}\left[\mathbf{x}_{n}\right]$. This module is among the most important representations in algebraic and geometric combinatorics. Its algebraic properties are closely tied to the combinatorics of permutations in $\mathfrak{S}_{n}$; let us recall some of these properties.

- The quotient $R_{n}$ has dimension $n$ ! as a $\mathbb{Q}$-vector space. In fact, E. Artin [2] used Galois theory to prove that the set of 'sub-staircase' monomials $\mathcal{A}_{n}:=$ $\left\{x_{1}^{i_{1}} \cdots x_{n}^{i_{n}}: 0 \leqslant i_{j}<j\right\}$ descends to a basis for $R_{n}$.

- A different monomial basis $\mathcal{G S}_{n}$ of $R_{n}$ was discovered by Garsia and Stanton [12]. Given a permutation $w=w(1) \ldots w(n) \in \mathfrak{S}_{n}$, the corresponding GS monomial basis element is

$$
g s_{w}:=\prod_{w(i)>w(i+1)} x_{w(1)} \cdots x_{w(i)} .
$$

- Chevalley [8] proved that $R_{n}$ is isomorphic as an ungraded $\mathfrak{S}_{n}$-module to the regular representation $\mathbb{Q}\left[\mathfrak{S}_{n}\right]$.

- Lusztig (unpublished) and Stanley described the graded $\mathfrak{S}_{n}$-module structure of $R_{n}$ using the major index statistic on standard Young tableaux [25].

Let $k \leqslant n$ be two positive integers. Haglund, Rhoades, and Shimozono [16, Defn. 1.1] introduced the ideal $I_{n, k} \subseteq \mathbb{Q}\left[\mathbf{x}_{n}\right]$ with generators

$$
I_{n, k}:=\left\langle x_{1}^{k}, x_{2}^{k}, \ldots, x_{n}^{k}, e_{n}\left(\mathbf{x}_{n}\right), e_{n-1}\left(\mathbf{x}_{n}\right), \ldots, e_{n-k+1}\left(\mathbf{x}_{n}\right)\right\rangle
$$

and studied the corresponding quotient ring $R_{n, k}:=\mathbb{Q}\left[\mathbf{x}_{n}\right] / I_{n, k}$. Since $I_{n, k}$ is homogeneous and stable under the action of $\mathfrak{S}_{n}$, the ring $R_{n, k}$ is a graded $\mathfrak{S}_{n}$-module. When $k=n$, we have $I_{n, n}=I_{n}$, so that $R_{n, n}=R_{n}$ and we recover the usual invariant ideal and coinvariant algebra.

To study $R_{n, k}$ one needs the notion of an ordered set partition of $[n]:=\{1,2, \ldots, n\}$, which is a set partition of $[n]$ with a total order on its blocks. For example, we have an ordered set partition

$$
\sigma=(25|6| 134)
$$

written in the 'bar notation'. The three blocks $\{2,5\},\{6\}$, and $\{1,3,4\}$ are ordered from left to right, and elements of each block are increasing.

Let $\mathcal{O P}{ }_{n, k}$ denote the collection of ordered set partitions of $[n]$ with $k$ blocks. We have

$$
\left|\mathcal{O} \mathcal{P}_{n, k}\right|=k ! \cdot \operatorname{Stir}(n, k),
$$

where $\operatorname{Stir}(n, k)$ is the (signless) Stirling number of the second kind counting $k$-block set partitions of $[n]$. The symmetric group $\mathfrak{S}_{n}$ acts on $\mathcal{O} \mathcal{P}_{n, k}$ by permuting the letters $1, \ldots, n$. For example, the permutation $w=241365$, written in one-line notation, sends $(25|6| 134)$ to $(46|5| 123)$.

Just as the structure of the classical coinvariant module $R_{n}$ is controlled by permutations in $\mathfrak{S}_{n}$, the structure of $R_{n, k}$ is governed by the collection $\mathcal{O} \mathcal{P}_{n, k}$ of ordered set partitions of $[n]$ with $k$ blocks [16].

- The dimension of $R_{n, k}$ is $\left|\mathcal{O} \mathcal{P}_{n, k}\right|=k$ ! $\cdot \operatorname{Stir}(n, k)$ [16, Thm. 4.11]. We have a generalization $\mathcal{A}_{n, k}$ of the Artin monomial basis to $R_{n, k}$ [16, Thm. 4.13].

- There is a generalization $\mathcal{G S}_{n, k}$ of the Garsia-Stanton monomial basis to $R_{n, k}$ [16, Thm. 5.3].

- The module $R_{n, k}$ is isomorphic as an ungraded $\mathfrak{S}_{n}$-representation to $\mathcal{O} \mathcal{P}_{n, k}$ [16, Thm. 4.11].

- There are explicit descriptions of the graded $\mathfrak{S}_{n}$-module structure of $R_{n, k}$ which generalize the work of Lusztig-Stanley [16, Thm 6.11, Cor. 6.12, Cor. 6.13, Thm. 6.14]. 
Now let $\mathbb{F}$ be an arbitrary field and let $n$ be a positive integer. The (type A) 0-Hecke algebra $H_{n}(0)$ is the unital associative $\mathbb{F}$-algebra with generators $\pi_{1}, \pi_{2}, \ldots, \pi_{n-1}$ and relations

$$
\begin{cases}\pi_{i}^{2}=\pi_{i} & 1 \leqslant i \leqslant n-1 \\ \pi_{i} \pi_{j}=\pi_{j} \pi_{i} & |i-j|>1, \\ \pi_{i} \pi_{i+1} \pi_{i}=\pi_{i+1} \pi_{i} \pi_{i+1} & 1 \leqslant i \leqslant n-2 .\end{cases}
$$

Recall that the symmetric group $\mathfrak{S}_{n}$ has Coxeter generators $\left\{s_{1}, s_{2}, \ldots, s_{n-1}\right\}$, where $s_{i}$ is the adjacent transposition $s_{i}=(i, i+1)$. These generators satisfy similar relations as (5) except that $s_{i}^{2}=1$ for all $i$. If $w \in \mathfrak{S}_{n}$ is a permutation and $w=s_{i_{1}} \cdots s_{i_{\ell}}$ is a reduced (i.e., as short as possible) expression for $w$ in the Coxeter generators $\left\{s_{1}, \ldots, s_{n-1}\right\}$, we define the 0 -Hecke algebra element $\pi_{w}:=\pi_{i_{1}} \cdots \pi_{i_{\ell}} \in H_{n}(0)$. It can be shown that the set $\left\{\pi_{w}: w \in \mathfrak{S}_{n}\right\}$ forms a basis for $H_{n}(0)$ as an $\mathbb{F}$-vector space, and in particular $H_{n}(0)$ has dimension $n$ !. In contrast to the situation with the symmetric group, the representation theory of the 0-Hecke algebra is insensitive to the choice of ground field, which motivates our generalization from $\mathbb{Q}$ to $\mathbb{F}$.

The algebra $H_{n}(0)$ is a deformation of the symmetric group algebra $\mathbb{F}\left[\mathfrak{S}_{n}\right]$. Roughly speaking, whereas in a typical $\mathbb{F}\left[\mathfrak{S}_{n}\right]$-module the generator $s_{i}$ acts by 'swapping' the letters $i$ and $i+1$, in a typical $H_{n}(0)$-module the generator $\pi_{i}$ acts by 'sorting' the letters $i$ and $i+1$. Indeed, the relations satisfied by the $\pi_{i}$ are precisely the relations satisfied by bubble sorting operators acting on a length $n$ list of entries $x_{1} \ldots x_{n}$ from a totally ordered alphabet:

$$
\pi_{i} \cdot\left(x_{1} \ldots x_{i} x_{i+1} \ldots x_{n}\right):= \begin{cases}x_{1} \ldots x_{i+1} x_{i} \ldots x_{n} & x_{i}>x_{i+1} \\ x_{1} \ldots x_{i} x_{i+1} \ldots x_{n} & x_{i} \leqslant x_{i+1}\end{cases}
$$

Proving 0-Hecke analogs of module theoretic results concerning the symmetric group has received a great deal of recent study in algebraic combinatorics $[4,17,18$, $26]$; let us recall the 0 -Hecke analog of the variable permutation action of $\mathfrak{S}_{n}$ on a polynomial ring.

Let $\mathbb{F}\left[\mathbf{x}_{n}\right]:=\mathbb{F}\left[x_{1}, \ldots, x_{n}\right]$ be the polynomial ring in $n$ variables over the field $\mathbb{F}$. The algebra $H_{n}(0)$ acts on $\mathbb{F}\left[\mathbf{x}_{n}\right]$ by the isobaric Demazure operators:

$$
\pi_{i}(f):=\frac{x_{i} f-x_{i+1}\left(s_{i}(f)\right)}{x_{i}-x_{i+1}}, \quad 1 \leqslant i \leqslant n-1 .
$$

If $f \in \mathbb{F}\left[\mathbf{x}_{n}\right]$ is symmetric in the variables $x_{i}$ and $x_{i+1}$, then $s_{i}(f)=f$ and thus $\pi_{i}(f)=$ $f$. The isobaric Demazure operators give a 0 -Hecke analog of variable permutation.

We also have a 0 -Hecke analog of the permutation action of $\mathfrak{S}_{n}$ on $\mathcal{O P}_{n, k}$. It is well-known that the 0 -Hecke algebra $H_{n}(0)$ has another generating set $\left\{\bar{\pi}_{1}, \ldots, \bar{\pi}_{n-1}\right\}$ subject to the relations

$$
\begin{cases}\bar{\pi}_{i}^{2}=-\bar{\pi}_{i} & 1 \leqslant i \leqslant n-1, \\ \bar{\pi}_{i} \bar{\pi}_{j}=\bar{\pi}_{j} \bar{\pi}_{i} & |i-j|>1, \\ \bar{\pi}_{i} \bar{\pi}_{i+1} \bar{\pi}_{i}=\bar{\pi}_{i+1} \bar{\pi}_{i} \bar{\pi}_{i+1} & 1 \leqslant i \leqslant n-2 .\end{cases}
$$

Here $\bar{\pi}_{i}:=\pi_{i}-1$ for all $i$. We will often use the relation $\bar{\pi}_{i} \pi_{i}=\pi_{i} \bar{\pi}_{i}=0$. One can define $\bar{\pi}_{w}:=\bar{\pi}_{i_{1}} \cdots \bar{\pi}_{i_{\ell}}$ for any $w \in \mathfrak{S}_{n}$ with a reduced expression $w=s_{i_{1}} \cdots s_{i_{\ell}}$ and show that the set $\left\{\bar{\pi}_{w}: w \in \mathfrak{S}_{n}\right\}$ is a basis for $H_{n}(0)$. Let $\mathbb{F}\left[\mathcal{O P} \mathcal{P}_{n, k}\right]$ be the $\mathbb{F}$-vector space with basis given by $\mathcal{O} \mathcal{P}_{n, k}$. Then $H_{n}(0)$ acts on $\mathbb{F}\left[\mathcal{O P}{ }_{n, k}\right]$ by the rule

$$
\bar{\pi}_{i} . \sigma:= \begin{cases}-\sigma, & \text { if } i+1 \text { appears in a block to the left of } i \text { in } \sigma, \\ s_{i}(\sigma), & \text { if } i+1 \text { appears in a block to the right of } i \text { in } \sigma, \\ 0, & \text { if } i+1 \text { appears in the same block as } i \text { in } \sigma,\end{cases}
$$


For example, we have

$$
\begin{aligned}
& \bar{\pi}_{1}(25|6| 134)=-(25|6| 134), \\
& \bar{\pi}_{2}(25|6| 134)=(35|6| 124), \\
& \bar{\pi}_{3}(25|6| 134)=0 .
\end{aligned}
$$

It is straightforward to check that these operators satisfy the relations (8) and so define an $H_{n}(0)$-action on $\mathbb{F}\left[\mathcal{O P} \mathcal{P}_{n, k}\right]$. In fact, this is a special case of an $H_{n}(0)$-action on generalized ribbon tableaux introduced in [18]. See also the proof of Lemma 5.2.

The coinvariant algebra $R_{n}$ can be viewed as a 0 -Hecke module. Indeed, the "Leibniz rule"

$$
\bar{\pi}_{i}(f g)=\bar{\pi}_{i}(f) g+s_{i}(f) \bar{\pi}_{i}(g)
$$

implies that the ideal $I_{n} \subseteq \mathbb{F}\left[\mathbf{x}_{n}\right]$ generated by $e_{1}\left(\mathbf{x}_{n}\right), \ldots, e_{n}\left(\mathbf{x}_{n}\right) \in \mathbb{F}\left[\mathbf{x}_{n}\right]$ is stable under the action of $H_{n}(0)$ on $\mathbb{F}\left[\mathbf{x}_{n}\right]$. Therefore, the quotient $R_{n}=\mathbb{F}\left[\mathbf{x}_{n}\right] / I_{n}$ inherits a 0 -Hecke action. Huang gave explicit formulas for its degree-graded and lengthdegree-bigraded quasisymmetric 0 -Hecke characteristic [17, Cor. 4.9]. The bivariant characteristic $\mathrm{Ch}_{q, t}\left(R_{n}\right)$ turns out to be a generating function for the pair of Mahonian statistics (inv, maj) on permutations in $\mathfrak{S}_{n}$, weighted by the Gessel fundamental quasisymmetric function $F_{\mathrm{iDes}(w)}$ corresponding to the inverse descent set iDes $(w)$ of $w \in \mathfrak{S}_{n}[17$, Cor. 4.9 (i)].

We will study a 0 -Hecke analog of the rings $R_{n, k}$ of Haglund, Rhoades, and Shimozono [16]. For $k<n$ the ideal $I_{n, k}$ is not usually stable under the action of $H_{n}(0)$ on $\mathbb{F}\left[\mathbf{x}_{n}\right]$, so that the quotient $\operatorname{ring} R_{n, k}=\mathbb{F}\left[\mathbf{x}_{n}\right] / I_{n, k}$ does not have the structure of an $H_{n}(0)$-module. To remedy this situation, we introduce the following modified family of ideals. Let

$$
h_{d}\left(x_{1}, \ldots, x_{i}\right):=\sum_{1 \leqslant j_{1} \leqslant \cdots \leqslant j_{d} \leqslant i} x_{j_{1}} \cdots x_{j_{d}}
$$

be the complete homogeneous symmetric function of degree $d$ in the variables $x_{1}, x_{2}, \ldots, x_{i}$.

DEFINITION 1.1. For two positive integers $k \leqslant n$, we define a quotient ring

$$
S_{n, k}:=\mathbb{F}\left[\mathbf{x}_{n}\right] / J_{n, k}
$$

where $J_{n, k} \subseteq \mathbb{F}\left[\mathbf{x}_{n}\right]$ is the ideal with generators

$J_{n, k}:=\left\langle h_{k}\left(x_{1}\right), h_{k}\left(x_{1}, x_{2}\right), \ldots, h_{k}\left(x_{1}, x_{2}, \ldots, x_{n}\right), e_{n}\left(\mathbf{x}_{n}\right), e_{n-1}\left(\mathbf{x}_{n}\right), \ldots, e_{n-k+1}\left(\mathbf{x}_{n}\right)\right\rangle$

The ideal $J_{n, k}$ is homogeneous. We claim that $J_{n, k}$ is stable under the action of $H_{n}(0)$. Since $e_{d}\left(\mathbf{x}_{n}\right) \in \mathbb{F}\left[\mathbf{x}_{n}\right]^{\mathfrak{S}_{n}}$ and $h_{k}\left(x_{1}, \ldots, x_{i}\right)$ is symmetric in $x_{j}$ and $x_{j+1}$ for $j \neq i$, thanks to Equation (10) this reduces to the observation that

$$
\pi_{i}\left(h_{k}\left(x_{1}, \ldots, x_{i}\right)\right)=h_{k}\left(x_{1}, \ldots, x_{i}, x_{i+1}\right) .
$$

Equation (12) is clear when $i=1$ and can be obtained from the following identity when $i \geqslant 2$ :

$$
h_{k}\left(x_{1}, \ldots, x_{i}\right)=\sum_{0 \leqslant j \leqslant k} x_{i}^{j} h_{k-j}\left(x_{1}, \ldots, x_{i-1}\right) .
$$

Thus the quotient $S_{n, k}$ has the structure of a graded $H_{n}(0)$-module.

It can be shown that $J_{n, n}=I_{n}$, so that $S_{n, n}=R_{n}$ is the classical coinvariant module. At the other extreme, we have $J_{n, 1}=\left\langle x_{1}, x_{2}, \ldots, x_{n}\right\rangle$, so that $S_{n, 1} \cong \mathbb{F}$ is the trivial $H_{n}(0)$-module in degree 0 .

Let us remark on an analogy between the generating sets of $I_{n, k}$ and $J_{n, k}$ which may rationalize the more complicated generating set of $J_{n, k}$. The defining representation 
of $\mathfrak{S}_{n}$ on $[n]$ is (of course) given by $s_{i}(i)=i+1, s_{i}(i+1)=i$, and $s_{i}(j)=j$ otherwise. The generators of $I_{n, k}$ come in two flavors:

(1) high degree elementary invariants $e_{n}\left(\mathbf{x}_{n}\right), e_{n-1}\left(\mathbf{x}_{n}\right), \ldots, e_{n-k+1}\left(\mathbf{x}_{n}\right)$, and

(2) a homogeneous system of parameters $\left\{x_{1}^{k}, x_{2}^{k}, \ldots, x_{n}^{k}\right\}$ of degree $k$ whose linear span is stable under the action of $\mathfrak{S}_{n}$ and isomorphic to the defining representation.

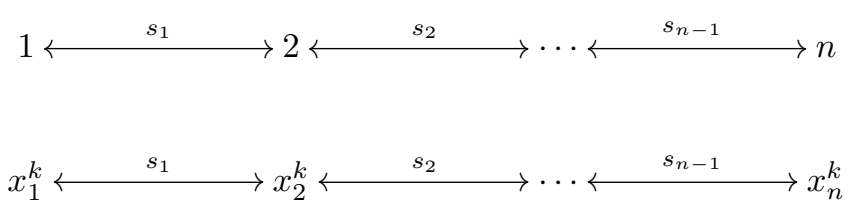

The defining representation of $H_{n}(0)$ on $[n]$ is given by $\pi_{i}(i)=i+1$ and $\pi_{i}(j)=j$ otherwise (whereas $s_{i}$ acts by swapping at $i, \pi_{i}$ acts by shifting at $i$ ). The generators of $J_{n, k}$ come in two analogous flavors:

(1) high degree elementary invariants $e_{n}\left(\mathbf{x}_{n}\right), e_{n-1}\left(\mathbf{x}_{n}\right), \ldots, e_{n-k+1}\left(\mathbf{x}_{n}\right)$, and

(2) a homogeneous system of parameters $\left\{h_{k}\left(x_{1}\right), \ldots, h_{k}\left(x_{1}, x_{2}, \ldots, x_{n}\right)\right\}$ of degree $k$ whose linear span is stable under the action of $H_{n}(0)$ and isomorphic to the defining representation (see (12)).

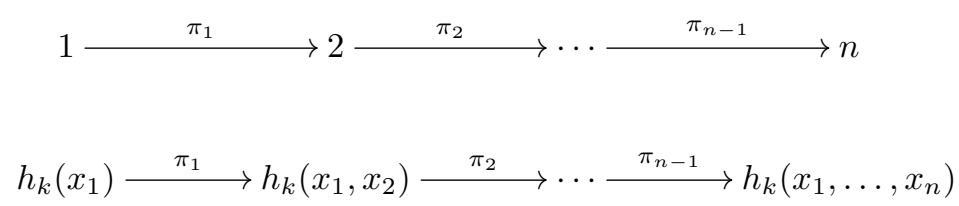

Deferring various definitions to Section 2, let us state our main results on $S_{n, k}$.

- The module $S_{n, k}$ has dimension $\left|\mathcal{O P}_{n, k}\right|=k$ ! $\cdot \operatorname{Stir}(n, k)$ as an $\mathbb{F}$-vector space (Theorem 3.8). There is a basis $\mathcal{C}_{n, k}$ for $S_{n, k}$, generalizing the Artin monomial basis of $R_{n}$. (Theorem 3.5, Corollary 3.6).

- There is a generalization $\mathcal{G S}_{n, k}$ of the the Garsia-Stanton monomial basis to $S_{n, k}$ (Corollary 4.3).

- As an ungraded $H_{n}(0)$-module, the quotient $S_{n, k}$ is isomorphic to $\mathbb{F}\left[\mathcal{O P}{ }_{n, k}\right]$ (Theorem 5.9).

- As a graded $H_{n}(0)$-module, we have explicit formulas for the degree-graded characteristics $\mathrm{Ch}_{t}\left(S_{n, k}\right)$ and $\mathbf{c h}_{t}\left(S_{n, k}\right)$ and the length-degree-bigraded characteristic $\mathrm{Ch}_{q, t}\left(S_{n, k}\right)$ of $S_{n, k}$ (Theorem 6.2, Corollary 6.4). The degree-graded quasisymmetric characteristic $\mathrm{Ch}_{t}\left(S_{n, k}\right)$ is symmetric and coincides with the graded Frobenius character of the $\mathfrak{S}_{n}$-module $R_{n, k}$ (over $\mathbb{Q}$ ).

The remainder of the paper is structured as follows. In Section 2 we give background and definitions related to compositions, ordered set partitions, Gröbner theory, and the representation theory of 0 -Hecke algebras. In Section 3 we will prove that the quotient $S_{n, k}$ has dimension $\left|\mathcal{O} \mathcal{P}_{n, k}\right|$ as an $\mathbb{F}$-vector space. We will derive a formula for the Hilbert series of $S_{n, k}$ and give a generalization of the Artin monomial basis to $S_{n, k}$. In Section 4 we will introduce a family of bases of $S_{n, k}$ which are related to the classical Garsia-Stanton basis in a unitriangular way when $k=n$. In Section 5 we will use one particular basis from this family to prove that the ungraded 0-Hecke structure of $S_{n, k}$ coincides with $\mathbb{F}\left[\mathcal{O P}{ }_{n, k}\right]$. In Section 6 we derive formulas for the degreegraded quasisymmetric and noncommutative symmetric characteristics $\operatorname{Ch}_{t}\left(S_{n, k}\right)$ and $\mathbf{c h}_{t}\left(S_{n, k}\right)$, and the length-degree-bigraded quasisymmetric characteristics $\mathrm{Ch}_{q, t}\left(S_{n, k}\right)$ of $S_{n, k}$. In Section 7 we make closing remarks. 


\section{BACKGROUND}

2.1. Compositions. Let $n$ be a nonnegative integer. A (strong) composition $\alpha$ of $n$ is a sequence $\alpha=\left(\alpha_{1}, \ldots, \alpha_{\ell}\right)$ of positive integers with $\alpha_{1}+\cdots+\alpha_{\ell}=n$. We call $\alpha_{1}, \ldots, \alpha_{\ell}$ the parts of $\alpha$. We write $\alpha=n$ to mean that $\alpha$ is a composition of $n$. We also write $|\alpha|=n$ for the size of $\alpha$ and $\ell(\alpha)=\ell$ for the number of parts of $\alpha$.

The descent set $\operatorname{Des}(\alpha)$ of a composition $\alpha=\left(\alpha_{1}, \ldots, \alpha_{\ell}\right) \models n$ is the subset of $[n-1]$ given by

$$
\operatorname{Des}(\alpha):=\left\{\alpha_{1}, \alpha_{1}+\alpha_{2}, \ldots, \alpha_{1}+\alpha_{2}+\cdots+\alpha_{\ell-1}\right\} .
$$

The map $\alpha \mapsto \operatorname{Des}(\alpha)$ gives a bijection from the set of compositions of $n$ to the collection of subsets of $[n-1]$. The major index of $\alpha=\left(\alpha_{1}, \ldots, \alpha_{\ell}\right)$ is

$$
\operatorname{maj}(\alpha):=\sum_{i \in \operatorname{Des}(\alpha)} i=(\ell-1) \cdot \alpha_{1}+\cdots+1 \cdot \alpha_{\ell-1}+0 \cdot \alpha_{\ell} .
$$

Given two compositions $\alpha, \beta \models n$, we write $\alpha \preceq \beta$ if $\operatorname{Des}(\alpha) \subseteq \operatorname{Des}(\beta)$. Equivalently, we have $\alpha \preceq \beta$ if the composition $\alpha$ can be formed by merging adjacent parts of the composition $\beta$. If $\alpha \models n$, the complement $\alpha^{c} \models n$ of $\alpha$ is the unique composition of $n$ which satisfies $\operatorname{Des}\left(\alpha^{c}\right)=[n-1] \backslash \operatorname{Des}(\alpha)$.

As an example of these concepts, let $\alpha=(2,3,1,2) \models 8$. We have $\ell(\alpha)=4$. The descent set of $\alpha$ is $\operatorname{Des}(\alpha)=\{2,5,6\}$. The major index is maj $(\alpha)=2+5+6=$ $3 \cdot 2+2 \cdot 3+1 \cdot 1+0 \cdot 2=13$. The complement of $\alpha$ is $\alpha^{c}=(1,2,1,3,1) \models 8$ with descent set $\operatorname{Des}\left(\alpha^{c}\right)=\{1,3,4,7\}=[7] \backslash\{2,5,6\}$.

If $\mathbf{i}=\left(i_{1}, \ldots, i_{n}\right)$ is any sequence of integers, the descent set Des(i) is given by

$$
\operatorname{Des}(\mathbf{i}):=\left\{1 \leqslant j \leqslant n-1: i_{j}>i_{j+1}\right\} .
$$

The descent number of $\mathbf{i}$ is $\operatorname{des}(\mathbf{i}):=|\operatorname{Des}(\mathbf{i})|$ and the major index of $\mathbf{i}$ is maj(i) $:=$ $\sum_{j \in \operatorname{Des}(\mathbf{i})} j$. Finally, the inversion number $\operatorname{inv}(\mathbf{i})$

$$
\operatorname{inv}(\mathbf{i}):=\left|\left\{\left(j, j^{\prime}\right): 1 \leqslant j<j^{\prime} \leqslant n, i_{j}>i_{j^{\prime}}\right\}\right|
$$

counts the number of inversion pairs in the sequence $\mathbf{i}$.

If a permutation $w \in \mathfrak{S}_{n}$ has one-line notation $w=w(1) \cdots w(n)$, we define $\operatorname{Des}(w), \operatorname{maj}(w), \operatorname{des}(w)$, and $\operatorname{inv}(w)$ as in the previous paragraph for the sequence $(w(1), \ldots, w(n))$. It turns out that $\operatorname{inv}(w)$ is equal to the Coxeter length $\ell(w)$ of $w$, i.e., the length of a reduced expression for $w$ in the generating set $\left\{s_{1}, \ldots, s_{n-1}\right\}$ of $\mathfrak{S}_{n}$. Moreover, we have $i \in \operatorname{Des}(w)$ if and only if some reduced expression of $w$ ends with $s_{i}$. We also let $i \operatorname{Des}(w):=\operatorname{Des}\left(w^{-1}\right)$ be the descent set of the inverse of the permutation $w$.

The statistics maj and inv are equidistributed on $\mathfrak{S}_{n}$ and their common distribution has a nice form. Let us recall the standard $q$-analogs of numbers, factorials, and multinomial coefficients:

$$
\begin{aligned}
& {[n]_{q}:=1+q+\cdots+q^{n-1}} \\
& {\left[\begin{array}{c}
n \\
a_{1}, \ldots, a_{r}
\end{array}\right]_{q}:=\frac{[n] !_{q}}{\left[a_{1}\right] !_{q} \cdots\left[a_{r}\right] !_{q}}} \\
& \begin{array}{r}
{[n] !_{q}:=[n]_{q}[n-1]_{q} \cdots[1]_{q}} \\
{\left[\begin{array}{l}
n \\
a
\end{array}\right]_{q}:=\frac{[n] !_{q}}{[a] !_{q}[n-a] !_{q}} .}
\end{array}
\end{aligned}
$$

MacMahon [20] proved

$$
\sum_{w \in \mathfrak{S}_{n}} q^{\operatorname{inv}(w)}=\sum_{w \in \mathfrak{S}_{n}} q^{\operatorname{maj}(w)}=[n] !_{q},
$$

and any statistic on $\mathfrak{S}_{n}$ which shares this distribution is called Mahonian. The joint distribution $\sum_{w \in \mathfrak{S}_{n}} q^{\operatorname{inv}(w)} t^{\operatorname{maj}(w)}$ of the pair of statistics (inv, maj) is called the biMahonian distribution. 
If $\alpha \models n$ and $\mathbf{i}=\left(i_{1}, \ldots, i_{n}\right)$ is a sequence of integers of length $n$, we define $\alpha \cup \mathbf{i} \models n$ to be the unique composition of $n$ which satisfies

$$
\operatorname{Des}(\alpha \cup \mathbf{i})=\operatorname{Des}(\alpha) \cup \operatorname{Des}(\mathbf{i}) .
$$

For example, let $\alpha=(3,2,3) \models 8$ and let $\mathbf{i}=(4,5,0,0,1,0,2,2)$. We have

$$
\operatorname{Des}(\alpha \cup \mathbf{i})=\operatorname{Des}(\alpha) \cup \operatorname{Des}(\mathbf{i})=\{3,5\} \cup\{2,5\}=\{2,3,5\},
$$

so that $\alpha \cup \mathbf{i}=(2,1,2,3)$. Whenever $\alpha \models n$ and $\mathbf{i}$ is a length $n$ sequence, we have the relation $\alpha \preceq \alpha \cup \mathbf{i}$.

A partition $\lambda$ of $n$ is a weakly decreasing sequence $\lambda=\left(\lambda_{1} \geqslant \cdots \geqslant \lambda_{\ell}\right)$ of positive integers which satisfies $\lambda_{1}+\cdots+\lambda_{\ell}=n$. We write $\lambda \vdash n$ to mean that $\lambda$ is a partition of $n$. We also write $|\lambda|=n$ for the size of $\lambda$ and $\ell(\lambda)=\ell$ for the number of parts of $\lambda$. The (English) Ferrers diagram of $\lambda$ consists of $\lambda_{i}$ left justified boxes in row $i$.

Identifying partitions with Ferrers diagrams, if $\mu \subseteq \lambda$ are a pair of partitions related by containment, the skew partition $\lambda / \mu$ is obtained by removing $\mu$ from $\lambda$. We write $|\lambda / \mu|:=|\lambda|-|\mu|$ for the number of boxes in this skew diagram. For example, the Ferrers diagrams of $\lambda$ and $\lambda / \mu$ are shown below, where $\lambda=(4,4,2)$ and $\mu=(2,1)$.
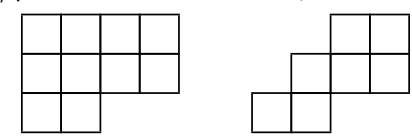

A semistandard tableau of a skew shape $\lambda / \mu$ is a filling of the Ferrers diagram of $\lambda / \mu$ with positive integers which are weakly increasing across rows and strictly increasing down columns. A standard tableau of shape $\lambda / \mu$ is a bijective filling of the Ferrers diagram of $\lambda / \mu$ with the numbers $1,2, \ldots,|\lambda / \mu|$ which is semistandard. An example of a semistandard tableau and a standard tableau of shape $(4,4,2) /(2,1)$ are shown below.
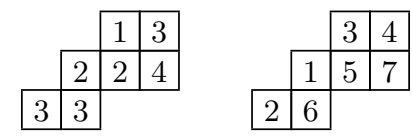

A ribbon is an edgewise connected skew diagram which contains no $2 \times 2$ square. The set of compositions of $n$ is in bijective correspondence with the set of size $n$ ribbons: a composition $\alpha=\left(\alpha_{1}, \ldots, \alpha_{\ell}\right)$ corresponds to the ribbon whose $i^{\text {th }}$ row from the bottom contains $\alpha_{i}$ boxes. We will identify compositions with ribbons in this way. For example, the ribbon corresponding to $\alpha=(2,3,1)$ is shown on the left below.
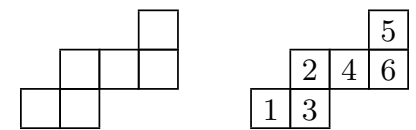

Let $\alpha \models n$ be a composition. We define a permutation $w_{0}(\alpha) \in \mathfrak{S}_{n}$ as follows. Starting at the leftmost column and working towards the right, and moving from top to bottom within each column, fill the ribbon diagram of $\alpha$ with the numbers $1,2, \ldots, n$ (giving a standard tableau). The permutation $w_{0}(\alpha)$ has one-line notation obtained by reading along the ribbon from the bottom row to the top row, proceeding from left to right within each row. It can be shown that $w_{0}(\alpha)$ is the unique left weak Bruhat minimal permutation $w \in \mathfrak{S}_{n}$ which satisfies $\operatorname{Des}(w)=\operatorname{Des}(\alpha)$ (cf. Björner and Wachs [6]). For example, if $\alpha=(2,3,1)$, the figure on the above right shows $w_{0}(\alpha)=132465 \in \mathfrak{S}_{6}$.

2.2. ORDERED SET PARTITIONS. As explained in Section 1, an ordered set partition $\sigma$ of size $n$ is a set partition of $[n]$ with a total order on its blocks. Let $\mathcal{O P} \mathcal{P}_{n, k}$ denote the collection of ordered set partitions of size $n$ with $k$ blocks. In particular, we may identify $\mathcal{O} \mathcal{P}_{n, n}$ with $\mathfrak{S}_{n}$. 
Also as in Section 1, we write an ordered set partition of $[n]$ as a permutation of $[n]$ with bars to separate blocks, such that letters within each block are increasing and blocks are ordered from left to right. For example, we have

$$
\sigma=(245|6| 13) \in \mathcal{O P}_{6,3} .
$$

The shape of an ordered set partition $\sigma=\left(B_{1}|\cdots| B_{k}\right)$ is the composition $\alpha=$ $\left(\left|B_{1}\right|, \ldots,\left|B_{k}\right|\right)$. For example, the above ordered set partition has shape $(3,1,2) \models 6$.

If $\alpha \models n$ is a composition, let $\mathcal{O P} \mathcal{P}_{\alpha}$ denote the collection of ordered set partitions of $n$ with shape $\alpha$. Given an ordered set partition $\sigma \in \mathcal{O P} \mathcal{P}_{\alpha}$, we can also represent $\sigma$ as the pair $(w, \alpha)$, where $w=w(1) \cdots w(n)$ is the permutation in $\mathfrak{S}_{n}$ (in oneline notation) obtained by erasing the bars in $\sigma$. For example, the above ordered set partition becomes

$$
\sigma=(245613,(3,1,2)) .
$$

This notation establishes a bijection between $\mathcal{O} \mathcal{P}_{n, k}$ and pairs $(w, \alpha)$ where $\alpha \models n$ is a composition with $\ell(\alpha)=k$ and $w \in \mathfrak{S}_{n}$ is a permutation with $\operatorname{Des}(w) \subseteq \operatorname{Des}(\alpha)$.

We extend the statistic maj from permutations to ordered set partitions as follows. Let $\sigma=\left(B_{1}|\cdots| B_{k}\right) \in \mathcal{O P}_{n, k}$ be an ordered set partition represented as a pair $(w, \alpha)$ as above. We define the major index $\operatorname{maj}(\sigma)$ to be the statistic

$$
\operatorname{maj}(\sigma)=\operatorname{maj}(w, \alpha):=\operatorname{maj}(w)+\sum_{i: \max \left(B_{i}\right)<\min \left(B_{i+1}\right)}\left(\alpha_{1}+\cdots+\alpha_{i}-i\right) .
$$

For example, if $\sigma=(24|57| 136 \mid 8)$, then

$$
\operatorname{maj}(\sigma)=\operatorname{maj}(24571368)+(2-1)+(2+2+3-3)=4+1+4=9 .
$$

We caution the reader that our definition of maj is not equivalent to, or even equidistributed with, the corresponding statistics for ordered set partitions in $[22,16]$ and elsewhere. However, the distribution of our maj on $\mathcal{O P}{ }_{n, k}$ is the reversal of the distribution of their maj.

The generating function for maj on $\mathcal{O P}_{n, k}$ may be described as follows. Let $\mathrm{rev}_{q}$ be the operator on polynomials in the variable $q$ which reverses coefficient sequences. For example, we have

$$
\operatorname{rev}_{q}\left(3 q^{3}+2 q^{2}+1\right)=q^{3}+2 q+3
$$

The $q$-Stirling number $\operatorname{Stir}_{q}(n, k)$ is defined by the recursion

$$
\operatorname{Stir}_{q}(n, k)=\operatorname{Stir}_{q}(n-1, k-1)+[k]_{q} \cdot \operatorname{Stir}_{q}(n-1, k)
$$

and the initial condition $\operatorname{Stir}_{q}(0, k)=\delta_{0, k}$, where $\delta$ is the Kronecker delta.

Proposition 2.1. Let $k \leqslant n$ be positive integers. We have

$$
\sum_{\sigma \in \mathcal{O P}} q^{\operatorname{maj}(\sigma)}=\operatorname{rev}_{q}\left([k] !_{q} \cdot \operatorname{Stir}_{q}(n, k)\right) .
$$

Proof. To see why this equation holds, consider the statistic maj' on an ordered set partition $\sigma=\left(B_{1}|\cdots| B_{k}\right)=(w, \alpha) \in \mathcal{O} \mathcal{P}_{n, k}$ defined by

$$
\operatorname{maj}^{\prime}(\sigma)=\operatorname{maj}^{\prime}(w, \alpha):=\sum_{i=1}^{k}(i-1)\left(\alpha_{i}-1\right)+\sum_{i: \min \left(B_{i}\right)>\max \left(B_{i+1}\right)} i .
$$

This is precisely the version of major index on ordered set partitions studied by Remmel and Wilson [22]. They proved [22, Eqn. 15, Prop. 5.1.1] that

$$
\sum_{\sigma \in \mathcal{O P}} q_{n, k}^{\text {maj'}^{\prime}(\sigma)}=[k] !_{q} \cdot \operatorname{Stir}_{q}(n, k) .
$$


On the other hand, for any $\sigma=(w, \alpha)=\left(B_{1}|\cdots| B_{k}\right) \in \mathcal{O} \mathcal{P}_{n, k}$ we have

$$
\operatorname{maj}(w)=\sum_{i: \max \left(B_{i}\right)>\min \left(B_{i+1}\right)}\left(\alpha_{1}+\cdots+\alpha_{i}\right) .
$$

This implies

$$
\begin{aligned}
\operatorname{maj}(\sigma) & =\operatorname{maj}(w)+\sum_{i: \max \left(B_{i}\right)<\min \left(B_{i+1}\right)}\left(\alpha_{1}+\cdots+\alpha_{i}-i\right) \\
& =\sum_{i=1}^{k-1}\left[(k-i) \cdot \alpha_{i}\right]-\sum_{i: \max \left(B_{i}\right)<\min \left(B_{i+1}\right)} i .
\end{aligned}
$$

The longest element $w_{0}=n \ldots 21$ (in one-line notation) of $\mathfrak{S}_{n}$ gives an involution on $\mathcal{O P}{ }_{\alpha}$ by

$$
\sigma=\left(B_{1}|\cdots| B_{k}\right) \mapsto w_{0}(\sigma)=\left(w_{0}\left(B_{1}\right)|\cdots| w_{0}\left(B_{k}\right)\right) .
$$

If $\alpha=n$ and $\ell(\alpha)=k$, then for $\sigma=\left(B_{1}|\cdots| B_{k}\right) \in \mathcal{O P} \mathcal{P}_{\alpha}$ and any index $1 \leqslant i \leqslant$ $k-1$ we have $\max \left(B_{i}\right)<\min \left(B_{i+1}\right)$ if and only if $\min \left(w_{0}\left(B_{i}\right)\right)>\max \left(w_{0}\left(B_{i+1}\right)\right)$. Therefore,

$$
\begin{aligned}
\operatorname{maj}^{\prime}(\sigma)+\operatorname{maj}\left(w_{0}(\sigma)\right) & =\sum_{i=1}^{k}\left[(i-1)\left(\alpha_{i}-1\right)+(k-i) \cdot \alpha_{i}\right] \\
& =\sum_{i=1}^{k}\left[-\alpha_{i}-i+1+k \alpha_{i}\right] \\
& =(k-1)(n-k)+\left(\begin{array}{l}
k \\
2
\end{array}\right) .
\end{aligned}
$$

On the other hand, it is easy to see that

$$
\max \left\{\operatorname{maj}(\sigma): \sigma \in \mathcal{O} \mathcal{P}_{n, k}\right\}=(k-1)(n-k)-\left(\begin{array}{l}
k \\
2
\end{array}\right)=\max \left\{\operatorname{maj}^{\prime}(\sigma): \sigma \in \mathcal{O P}_{n, k}\right\}
$$

Applying Equation (24) gives

$$
\sum_{\sigma \in \mathcal{O P}_{n, k}} q^{\operatorname{maj}(\sigma)}=\operatorname{rev}_{q}\left[\sum_{\sigma \in \mathcal{O P}} q_{n, k}^{\operatorname{maj}^{\prime}(\sigma)}\right]=\operatorname{rev}_{q}\left([k] !_{q} \cdot \operatorname{Stir}_{q}(n, k)\right) .
$$

We have an action of the 0 -Hecke algebra $H_{n}(0)$ on $\mathbb{F}\left[\mathcal{O P}{ }_{n, k}\right]$ given by Equation (9). This $H_{n}(0)$-action preserves $\mathbb{F}\left[\mathcal{O P} \mathcal{P}_{\alpha}\right]$ for each composition $\alpha$ of $n$.

2.3. GröBner THEORY. We review material related to Gröbner bases of ideals $I \subseteq$ $\mathbb{F}\left[\mathbf{x}_{n}\right]$ and standard monomial bases of the corresponding quotients $\mathbb{F}\left[\mathbf{x}_{n}\right] / I$. For a more leisurely introduction to this material, see [9].

A total order $\leqslant$ on the monomials in $\mathbb{F}\left[\mathbf{x}_{n}\right]$ is called a term order if

- $1 \leqslant m$ for all monomials $m \in \mathbb{F}\left[\mathbf{x}_{n}\right]$, and

- if $m, m^{\prime}, m^{\prime \prime} \in \mathbb{F}\left[\mathbf{x}_{n}\right]$ are monomials, then $m \leqslant m^{\prime}$ implies $m \cdot m^{\prime \prime} \leqslant m^{\prime} \cdot m^{\prime \prime}$.

In this paper, we will consider the lexicographic term order with respect to the variable ordering $x_{n}>\cdots>x_{2}>x_{1}$. That is, we have

$$
x_{1}^{a_{1}} \cdots x_{n}^{a_{n}}<x_{1}^{b_{1}} \cdots x_{n}^{b_{n}}
$$

if and only if there exists an integer $1 \leqslant j \leqslant n$ such that $a_{j+1}=b_{j+1}, \ldots, a_{n}=b_{n}$, and $a_{j}<b_{j}$. Following the notation of SAGE, we call this term order neglex. 
Let $\leqslant$ be any term order on monomials in $\mathbb{F}\left[\mathbf{x}_{n}\right]$. If $f \in \mathbb{F}\left[\mathbf{x}_{n}\right]$ is a nonzero polynomial, let $\operatorname{in}_{<}(f)$ be the leading (i.e., largest) term of $f$ with respect to $<$. If $I \subseteq \mathbb{F}\left[\mathbf{x}_{n}\right]$ is an ideal, the associated initial ideal is the monomial ideal

$$
\operatorname{in}_{<}(I):=\left\langle\operatorname{in}_{<}(f): f \in I-\{0\}\right\rangle .
$$

The set of monomials

$$
\left\{\text { monomials } m \in \mathbb{F}\left[\mathbf{x}_{n}\right]: m \notin \operatorname{in}_{<}(I)\right\}
$$

descends to a $\mathbb{F}$-basis for the quotient $\mathbb{F}\left[\mathbf{x}_{n}\right] / I$; this basis is called the standard monomial basis (with respect to the term order $\leqslant$ ) [9, Prop. 1, p. 230].

Let $I \subseteq \mathbb{F}\left[\mathbf{x}_{n}\right]$ be any ideal and let $\leqslant$ be a term order. A finite set $G=\left\{g_{1}, \ldots, g_{r}\right\} \subseteq$ $I$ of nonzero polynomials in $I$ is called a Gröbner basis of $I$ if

$$
\operatorname{in}_{<}(I)=\left\langle\operatorname{in}_{<}\left(g_{1}\right), \ldots, \text { in }_{<}\left(g_{r}\right)\right\rangle .
$$

If $G$ is a Gröbner basis of $I$, then we have $I=\langle G\rangle$ [9, Cor. 6, p. 77].

Let $G$ be a Gröbner basis for $I$ with respect to the term order $\leqslant$. The basis $G$ is called minimal if

- for any $g \in G$, the leading coefficient of $g$ with respect to $\leqslant$ is 1 , and

- for any $g \neq g^{\prime}$ in $G$, the leading monomial of $g$ does not divide the leading monomial of $g^{\prime}$.

A minimal Gröbner basis $G$ is called reduced if in addition

- for any $g \neq g^{\prime}$ in $G$, the leading monomial of $g$ does not divide any term in the polynomial $g^{\prime}$.

Up to a choice of term order, every ideal $I$ has a unique reduced Gröbner basis $[9$, Prop. 6, p. 92].

2.4. Sym, QSym, AND NSym. Let $\mathbf{x}=\left(x_{1}, x_{2}, \ldots\right)$ be a totally ordered infinite set of variables and let Sym be the $(\mathbb{Z}$-)algebra of symmetric functions in $\mathbf{x}$ with coefficients in $\mathbb{Z}$. The algebra Sym is graded; its degree $n$ component has basis given by the collection $\left\{s_{\lambda}: \lambda \vdash n\right\}$ of Schur functions. The Schur function $s_{\lambda}$ may be defined as

$$
s_{\lambda}=\sum_{T} \mathbf{x}^{T}
$$

where the sum is over all semistandard tableaux $T$ of shape $\lambda$ and $\mathbf{x}^{T}$ is the monomial

$$
\mathbf{x}^{T}:=x_{1}^{\# \text { of } 1 \mathrm{~s} \text { in } T} x_{2}^{\# \text { of } 2 \mathrm{~s} \text { in } T} \ldots .
$$

Given partitions $\mu \subseteq \lambda$, we also let $s_{\lambda / \mu} \in$ Sym denote the associated skew Schur function. The expansion of $s_{\lambda / \mu}$ in the $\mathbf{x}$ variables is also given by Equation (35). In particular, if $\alpha$ is a composition (thought of as a ribbon), we have the ribbon Schur function $s_{\alpha} \in$ Sym.

There is a coproduct of Sym given by replacing the variables $x_{1}, x_{2}, \ldots$ with $x_{1}, x_{2}, \ldots, y_{1}, y_{2}, \ldots$ such that Sym becomes a graded Hopf algebra which is self-dual under the basis $\left\{s_{\lambda}\right\}[14, \S 2]$.

Let $\alpha=\left(\alpha_{1}, \ldots, \alpha_{k}\right) \models n$ be a composition. The monomial quasisymmetric function is the formal power series

$$
M_{\alpha}:=\sum_{i_{1}<\cdots<i_{k}} x_{i_{1}}^{\alpha_{1}} \cdots x_{i_{k}}^{\alpha_{k}} .
$$

The graded algebra of quasisymmetric functions QSym is the $\mathbb{Z}$-linear span of the $M_{\alpha}$, where $\alpha$ ranges over all compositions. 
We will focus on a basis for QSym other than the monomial quasisymmetric functions $M_{\alpha}$. If $n$ is a positive integer and if $S \subseteq[n-1]$, the Gessel fundamental quasisymmetric function $F_{S}$ attached to $S$ is

$$
F_{S}:=\sum_{\substack{i_{1} \leqslant \cdots \leqslant i_{n} \\ j \in S \Rightarrow i_{j}<i_{j+1}}} x_{i_{1}} \cdots x_{i_{n}} .
$$

In particular, if $w \in \mathfrak{S}_{n}$ is a permutation with inverse descent set $\operatorname{iDes}(w) \subseteq[n-1]$, we have the quasisymmetric function $F_{\mathrm{iDes}(w)}$. If $\alpha \models n$ is a composition, we extend this notation by setting $F_{\alpha}:=F_{\operatorname{Des}(\alpha)}$.

Next, let NSym be the graded algebra of noncommutative symmetric functions. This is the free unital associative (noncommutative) algebra

$$
\text { NSym }:=\mathbb{Z}\left\langle\mathbf{h}_{1}, \mathbf{h}_{2}, \ldots\right\rangle
$$

generated over $\mathbb{Z}$ by the symbols $\mathbf{h}_{1}, \mathbf{h}_{2}, \ldots$, where $\mathbf{h}_{d}$ has degree $d$. The degree $n$ component of $\mathbf{N S y m}$ has $\mathbb{Z}$-basis given by $\left\{\mathbf{h}_{\alpha}: \alpha \models n\right\}$, where for $\alpha=\left(\alpha_{1}, \ldots, \alpha_{\ell}\right) \models n$ we set

$$
\mathbf{h}_{\alpha}:=\mathbf{h}_{\alpha_{1}} \cdots \mathbf{h}_{\alpha_{\ell}} .
$$

Another basis of the degree $n$ piece of NSym consists of the ribbon Schur functions $\left\{\mathbf{s}_{\alpha}: \alpha \models n\right\}$. The ribbon Schur function $\mathbf{s}_{\alpha}$ is defined by

$$
\mathbf{s}_{\alpha}:=\sum_{\beta \preceq \alpha}(-1)^{\ell(\alpha)-\ell(\beta)} \mathbf{h}_{\beta} .
$$

Finally, there are coproducts for QSym and NSym such that they become dual graded Hopf algebras [14, §5].

2.5. Characteristic maps. Let $A$ be a finite-dimensional algebra over a field $\mathbb{F}$. The Grothendieck group $G_{0}(A)$ of the category of finitely-generated A-modules is the quotient of the free abelian group generated by isomorphism classes $[M]$ of finitelygenerated $A$-modules $M$ by the subgroup generated by elements $[M]-[L]-[N]$ corresponding to short exact sequences $0 \rightarrow L \rightarrow M \rightarrow N \rightarrow 0$ of finitely-generated $A$-modules. The abelian group $G_{0}(A)$ has free basis given by the collection of (isomorphism classes of) irreducible $A$-modules. The Grothendieck group $K_{0}(A)$ of the category of finitely-generated projective A-modules is defined similarly, and has free basis given by the set of (isomorphism classes of) projective indecomposable $A$-modules. If $A$ is semisimple then $G_{0}(A)=K_{0}(A)$. See [3] for more details on representation theory of finite dimensional algebras.

The symmetric group algebra $\mathbb{Q}\left[\mathfrak{S}_{n}\right]$ is semisimple and has irreducible representations $S^{\lambda}$ indexed by partitions $\lambda \vdash n$. The Grothendieck group $G_{0}\left(\mathbb{Q}\left[\mathfrak{S}_{\bullet}\right]\right)$ of the tower $\mathbb{Q}\left[\mathfrak{S}_{.}\right]: \mathbb{Q}\left[\mathfrak{S}_{0}\right] \hookrightarrow \mathbb{Q}\left[\mathfrak{S}_{1}\right] \hookrightarrow \mathbb{Q}\left[\mathfrak{S}_{2}\right] \hookrightarrow \cdots$ of symmetric group algebras is the direct sum of $G_{0}\left(\mathbb{Q}\left[\mathfrak{S}_{n}\right]\right)$ for all $n \geqslant 0$. It is a graded Hopf algebra with product and coproduct given by induction and restriction along the embeddings $\mathfrak{S}_{m} \times \mathfrak{S}_{n} \hookrightarrow \mathfrak{S}_{m+n}$. The structure constants of $G_{0}\left(\mathbb{Q}\left[\mathfrak{S}_{\mathbf{\bullet}}\right]\right)$ under the self-dual basis $\left\{S^{\lambda}\right\}$, where $\lambda$ runs through all partitions, are the well-known Littlewood-Richardson coefficients.

The Frobenius character ${ }^{(1)} \operatorname{Frob}(V)$ of a finite-dimensional $\mathbb{Q}\left[\mathfrak{S}_{n}\right]$-module $V$ is

$$
\operatorname{Frob}(V):=\sum_{\lambda \vdash n}\left[V: S^{\lambda}\right] \cdot s_{\lambda} \in \operatorname{Sym}
$$

\footnotetext{
${ }^{(1)}$ The Frobenius character Frob $(V)$ is indeed a "character" since the Schur functions are characters of irreducible polynomial representations of the general linear groups.
} 
where $\left[V: S^{\lambda}\right]$ is the multiplicity of the simple module $S^{\lambda}$ among the composition factors of $V$. The correspondence Frob gives a graded Hopf algebra isomorphism $G_{0}\left(\mathbb{Q}\left[\mathfrak{S}_{\bullet}\right]\right) \cong \operatorname{Sym}[14, \S 4.4]$.

One can refine Frob for graded representations of $\mathbb{Q}\left[\mathfrak{S}_{n}\right]$. Recall that the Hilbert series of a graded vector space $V=\bigoplus_{d \geqslant 0} V_{d}$ with each component $V_{d}$ finite-dimensional is

$$
\operatorname{Hilb}(V ; q):=\sum_{d \geqslant 0} \operatorname{dim}\left(V_{d}\right) \cdot q^{d} .
$$

If $V$ carries a graded action of $\mathbb{Q}\left[\mathfrak{S}_{n}\right]$, we also define the graded Frobenius series by

$$
\operatorname{grFrob}(V ; q):=\sum_{d \geqslant 0} \operatorname{Frob}\left(V_{d}\right) \cdot q^{d} .
$$

Now let us recall the 0 -Hecke analog of the above story. Consider an arbitrary ground field $\mathbb{F}$. The representation theory of the $\mathbb{F}$-algebra $H_{n}(0)$ was studied by Norton [21] and has a different flavor from that of $\mathbb{Q}\left[\mathfrak{S}_{n}\right]$ since $H_{n}(0)$ is not semisimple. Norton [21] proved that the $H_{n}(0)$-modules

$$
P_{\alpha}:=H_{n}(0) \bar{\pi}_{w_{0}(\alpha)} \pi_{w_{0}\left(\alpha^{c}\right)},
$$

where $\alpha$ ranges over all compositions of $n$, form a complete list of nonisomorphic indecomposable projective $H_{n}(0)$-modules. For each $\alpha \models n$, the $H_{n}(0)$-module $P_{\alpha}$ has a basis

$$
\left\{\bar{\pi}_{w} \pi_{w_{0}\left(\alpha^{c}\right)}: w \in \mathfrak{S}_{n}, \operatorname{Des}(w)=\operatorname{Des}(\alpha)\right\} .
$$

Moreover, $P_{\alpha}$ has a unique maximal submodule spanned by all elements in the above basis except its cyclic generator $\bar{\pi}_{w_{0}(\alpha)} \pi_{w_{0}\left(\alpha^{c}\right)}$, and the quotient of $P_{\alpha}$ by this maximal submodule, denoted by $C_{\alpha}$, is one-dimensional and admits an $H_{n}(0)$-action by $\bar{\pi}_{i}=$ -1 for all $i \in \operatorname{Des}(\alpha)$ and $\bar{\pi}_{i}=0$ for all $i \in \operatorname{Des}\left(\alpha^{c}\right)$. The collections $\left\{P_{\alpha}: \alpha \models n\right\}$ and $\left\{C_{\alpha}: \alpha \models n\right\}$ are complete lists of nonisomorphic projective indecomposable and irreducible $H_{n}(0)$-modules, respectively.

Just as the Frobenius character map gives a deep connection between the representation theory of symmetric groups and the ring Sym of symmetric functions, there are two characteristic maps Ch and $\mathbf{c h}$, defined by Krob and Thibon [19], which facilitate the study of representations of $H_{n}(0)$ through the rings QSym and NSym. Let us recall their construction.

The two Grothendieck groups $G_{0}\left(H_{n}(0)\right)$ and $K_{0}\left(H_{n}(0)\right)$ have free $\mathbb{Z}$-bases $\left\{C_{\alpha}\right.$ : $\alpha \models n\}$ and $\left\{P_{\alpha}: \alpha \models n\right\}$, respectively. Associated to the tower of algebras $H_{\bullet}(0)$ : $H_{0}(0) \hookrightarrow H_{1}(0) \hookrightarrow H_{2}(0) \hookrightarrow \cdots$ are the two Grothendieck groups

$$
G_{0}\left(H_{\bullet}(0)\right):=\bigoplus_{n \geqslant 0} G_{0}\left(H_{n}(0)\right) \text { and } K_{0}\left(H_{\bullet}(0)\right):=\bigoplus_{n \geqslant 0} K_{0}\left(H_{n}(0)\right) .
$$

These groups are graded Hopf algebras with product and coproduct given by induction and restriction along the embeddings $H_{n}(0) \otimes H_{m}(0) \hookrightarrow H_{n+m}(0)$, and they are dual to each other via the pairing $\left\langle P_{\alpha}, C_{\beta}\right\rangle=\delta_{\alpha, \beta}$.

Analogously to the Frobenius correspondence, Krob and Thibon [19] defined two linear maps

$$
\mathrm{Ch}: G_{0}\left(H_{\bullet}(0)\right) \rightarrow \text { QSym and } \mathbf{c h}: K_{0}\left(H_{\bullet}(0)\right) \rightarrow \text { NSym }
$$

by $\operatorname{Ch}\left(C_{\alpha}\right):=F_{\alpha}$ and $\operatorname{ch}\left(P_{\alpha}\right):=\mathbf{s}_{\alpha}$ for all compositions $\alpha$. These maps are isomorphisms of graded Hopf algebras. Krob and Thibon also showed [19] that for any composition $\alpha$, the characteristic $\mathrm{Ch}\left(P_{\alpha}\right)$ equals the corresponding ribbon Schur function $s_{\alpha} \in$ Sym:

$$
\operatorname{Ch}\left(P_{\alpha}\right)=\sum_{w \in \mathfrak{S}_{n}: \operatorname{Des}(w)=\operatorname{Des}(\alpha)} F_{\mathrm{iDes}(w)}=s_{\alpha}
$$


We give graded extensions of the maps $\mathrm{Ch}$ and $\mathbf{c h}$ as follows. Suppose that $V=$ $\bigoplus_{d \geqslant 0} V_{d}$ is a graded $H_{n}(0)$-module with finite-dimensional homogeneous components $V_{d}$. The degree-graded noncommutative characteristic and degree-graded quasisymmetric characteristic of $V$ are defined by

$$
\operatorname{ch}_{t}(V):=\sum_{d \geqslant 0} \operatorname{ch}\left(V_{d}\right) \cdot t^{d} \quad \text { and } \quad \operatorname{Ch}_{t}(V):=\sum_{d \geqslant 0} \operatorname{Ch}\left(V_{d}\right) \cdot t^{d} .
$$

On the other hand, the 0-Hecke algebra $H_{n}(0)$ has a length filtration $H_{n}(0)^{(0)} \supseteq$ $H_{n}(0)^{(1)} \supseteq H_{n}(0)^{(2)} \supseteq \cdots$ where $H_{n}(0)^{(\ell)}$ is the span of $\left\{\pi_{w}: w \in \mathfrak{S}_{n}, \ell(w) \geqslant \ell\right\}$. Let $V=H_{n}(0) v$ be a cyclic $H_{n}(0)$-module whose distinguished generator $v \in V$ is equipped with a length $a \geqslant 0$. The length filtration $V^{(a)} \supseteq V^{(a+1)} \supseteq V^{(a+2)} \supseteq \cdots$ of $V$ is given by

$$
V^{(\ell)}:=H_{n}(0)^{(\ell-a)} v, \quad \ell \geqslant a .
$$

Following Krob and Thibon [19], we define the length-graded quasymmetric characteristic of $V$ as

$$
\mathrm{Ch}_{q}(V):=\sum_{\ell \geqslant a} \operatorname{Ch}\left(V^{(\ell)} / V^{(\ell+1)}\right) \cdot q^{\ell} .
$$

The freedom to choose a length $a \geqslant 0$ for the distinguished generator $v$ will make certain formulas look nicer.

Now suppose $V=\bigoplus_{d \geqslant 0} V_{d}$ is a graded $H_{n}(0)$-module which is also cyclic with a length filtration $V^{(a)} \supseteq V^{(a+1)} \supseteq \cdots$ as in the above paragraph. Let $V_{d}^{(\ell)}:=$ $V^{(\ell)} \cap V_{d}$ for $\ell \geqslant a$ and $\bar{d} \geqslant 0$. We define the length-degree-bigraded quasisymmetric characteristic of $V$ to be

$$
\mathrm{Ch}_{q, t}(V):=\sum_{\substack{\ell \geqslant a \\ d \geqslant 0}} \operatorname{Ch}\left(V_{d}^{(\ell)} / V_{d}^{(\ell+1)}\right) \cdot q^{\ell} t^{d} .
$$

Finally, if an $H_{n}(0)$-module $V=\bigoplus_{\alpha \in I} V_{\alpha}$ is a direct sum of cyclic graded $H_{n}(0)$ submodules $V_{\alpha}$ for $\alpha$ in some index set $I$, then we define $\mathrm{Ch}_{q, t}(V):=\sum_{\alpha \in I} \mathrm{Ch}_{q, t}\left(V_{\alpha}\right)$. Note that $\mathrm{Ch}_{q, t}(V)$ may depend on the choice of the direct sum decomposition of $V$ into cyclic submodules. For example, Huang [17] showed that the coinvariant algebra $R_{n}$ is isomorphic to the regular representation of $H_{n}(0)$ and obtained the lengthdegree-bigraded quasisymmetric characteristic

$$
\mathrm{Ch}_{q, t}\left(R_{n}\right)=\sum_{w \in \mathfrak{S}_{n}} q^{\operatorname{inv}(w)} t^{\operatorname{maj}(w)} F_{\mathrm{iDes}(w)}
$$

using the cyclic generator of $R_{n}$ corresponding to the element $1 \in H_{n}(0)$. However, if $R_{n}$ is viewed as a direct sum of projective indecomposable submodules indexed by compositions of $n$ then the length grading received by each $w \in \mathfrak{S}_{n}$ needs to be changed to $\operatorname{inv}(w)-\operatorname{inv}\left(w_{0}(\alpha)\right)$ where $\alpha \models n$ is determined by $\operatorname{Des}(\alpha)=\operatorname{Des}(w)$. For our convenience, we will choose an approriate decomposition of $V$ into cyclic submodules, and further adjust the length grading by a suitable constant for each cyclic submodule in the distinguished direct sum decomposition of $V$. This will give a length-degree-bigraded characteristic $\mathrm{Ch}_{q, t}(V)$, which specializes to $\mathrm{Ch}_{1, t}(V)=$ $\mathrm{Ch}_{t}(V)$ and $\mathrm{Ch}_{q, 1}(V)=\mathrm{Ch}_{q}(V)$, respectively.

\section{Hilbert Series and Artin basis}

3.1. The POINT SETS $Z_{n, k}$. In this section we will prove that $\operatorname{dim}\left(S_{n, k}\right)=\left|\mathcal{O} \mathcal{P}_{n, k}\right|$. To do this, we will use tools from elementary algebraic geometry. This basic method 
dates back to the work of Garsia and Procesi on Springer fibers and Tanisaki quotients [11].

Given a finite point set $Z \subseteq \mathbb{F}^{n}$, let $\mathbf{I}(Z) \subseteq \mathbb{F}\left[\mathbf{x}_{n}\right]$ be the ideal of polynomials which vanish on $Z$ :

$$
\mathbf{I}(Z):=\left\{f \in \mathbb{F}\left[\mathbf{x}_{n}\right]: f(\mathbf{z})=0 \text { for all } \mathbf{z} \in Z\right\} .
$$

There is a natural identification of the quotient $\mathbb{F}\left[\mathbf{x}_{n}\right] / \mathbf{I}(Z)$ with the collection of polynomial functions $Z \rightarrow \mathbb{F}$.

We claim that any function $Z \rightarrow \mathbb{F}$ may be realized as the restriction of a polynomial function. This essentially follows from Lagrange Interpolation. Indeed, since $Z \subseteq$ $\mathbb{F}^{n}$ is finite, there exist field elements $\alpha_{1}, \ldots, \alpha_{m} \in \mathbb{F}$ such that $Z \subseteq\left\{\alpha_{1}, \ldots, \alpha_{m}\right\}^{n}$. For any $n$-tuple of integers $\left(i_{1}, \ldots, i_{n}\right)$ between 1 and $m$, the polynomial

$$
\prod_{j_{1} \neq i_{1}}\left(x_{1}-\alpha_{j_{1}}\right) \cdots \prod_{j_{n} \neq i_{n}}\left(x_{n}-\alpha_{j_{n}}\right) \in \mathbb{F}\left[\mathbf{x}_{n}\right]
$$

vanishes on every point of $\left\{\alpha_{1}, \ldots, \alpha_{m}\right\}^{n}$ except for $\left(\alpha_{i_{1}}, \ldots, \alpha_{i_{n}}\right)$. Hence, an arbitrary $\mathbb{F}$-valued function on $\left\{\alpha_{1}, \ldots, \alpha_{m}\right\}^{n}$ may be realized using a linear combination of polynomials of the above form. Since $Z \subseteq\left\{\alpha_{1}, \ldots, \alpha_{m}\right\}^{n}$, the same is true for an arbitrary $\mathbb{F}$-valued function on $Z$.

By the last paragraph, we may identify the quotient $\mathbb{F}\left[\mathbf{x}_{n}\right] / \mathbf{I}(Z)$ with the collection of all functions $Z \rightarrow \mathbb{F}$. In particular, the dimension of this quotient as an $\mathbb{F}$-vector space is

$$
\operatorname{dim}\left(\mathbb{F}\left[\mathbf{x}_{n}\right] / \mathbf{I}(Z)\right)=|Z| .
$$

The ideal $\mathbf{I}(Z)$ is almost never homogeneous. To get a homogeneous ideal, we do the following. For any nonzero polynomial $f \in \mathbb{F}\left[\mathbf{x}_{n}\right]$, let $\tau(f)$ be the top degree component of $f$. That is, if $f=f_{d}+f_{d-1}+\cdots+f_{0}$ where $f_{i}$ has homogeneous degree $i$ for all $i$ and $f_{d} \neq 0$, then $\tau(f)=f_{d}$. The ideal $\mathbf{T}(Z) \subseteq \mathbb{F}\left[\mathbf{x}_{n}\right]$ is generated by the top degree components of all nonzero polynomials in $\mathbf{I}(Z)$. In symbols:

$$
\mathbf{T}(Z):=\langle\tau(f): f \in \mathbf{I}(Z)-\{0\}\rangle .
$$

The ideal $\mathbf{T}(Z)$ is homogeneous by definition, so that $\mathbb{F}\left[\mathbf{x}_{n}\right] / \mathbf{T}(Z)$ is a graded $\mathbb{F}$-vector space. Moreover, it is well known that

$$
\operatorname{dim}\left(\mathbb{F}\left[\mathbf{x}_{n}\right] / \mathbf{T}(Z)\right)=\operatorname{dim}\left(\mathbb{F}\left[\mathbf{x}_{n}\right] / \mathbf{I}(Z)\right)=|Z| .
$$

Our three-step strategy for proving $\operatorname{dim}\left(S_{n, k}\right)=\left|\mathcal{O} \mathcal{P}_{n, k}\right|$ is as follows.

(1) Find a point set $Z_{n, k} \subseteq \mathbb{F}^{n}$ which is in bijective correspondence with $\mathcal{O P} \mathcal{P}_{n, k}$.

(2) Prove that the generators of $J_{n, k}$ arise as top degree components of polynomials in $\mathbf{I}\left(Z_{n, k}\right)$, so that $J_{n, k} \subseteq \mathbf{T}\left(Z_{n, k}\right)$.

(3) Use Gröbner theory to prove $\operatorname{dim}\left(S_{n, k}\right) \leqslant\left|\mathcal{O P}_{n, k}\right|$, forcing $\operatorname{dim}\left(S_{n, k}\right)=$ $\left|\mathcal{O P} \mathcal{P}_{n, k}\right|$ by Steps 1 and 2 .

A similar three-step strategy was used by Haglund, Rhoades, and Shimozono [16] in their analysis of the $\mathfrak{S}_{n}$-module structure of $R_{n, k}$. In our setting, since we do not have a group action, we can only use this strategy to deduce the vector space structure of $S_{n, k}$, rather than the $H_{n}(0)$-module structure of $S_{n, k}$.

To achieve Step 1 of our strategy, we need to find a candidate set $Z_{n, k} \subseteq \mathbb{F}^{n}$ which is in bijective correspondence with $\mathcal{O P}_{n, k}$. Here we run into a problem: to define our candidate point sets, we need the field $\mathbb{F}$ to contain at least $n+k-1$ elements. This problem did not arise in the work of Haglund et. al. [16]; they worked exclusively over the field $\mathbb{Q}$. To get around this problem, we use the following trick. 
LEMmA 3.1. Let $\mathbb{F} \subseteq \mathbb{K}$ be a field extension and $J=\left\langle f_{1}, \ldots, f_{r}\right\rangle \subseteq \mathbb{F}\left[\mathbf{x}_{n}\right]$ an ideal of $\mathbb{F}\left[\mathbf{x}_{n}\right]$ generated by $f_{1}, \ldots, f_{r} \in \mathbb{F}\left[\mathbf{x}_{n}\right]$. Then $\operatorname{dim}_{\mathbb{F}}\left(\mathbb{F}\left[\mathbf{x}_{n}\right] / J\right)=\operatorname{dim}_{\mathbb{K}}\left(\mathbb{K}\left[\mathbf{x}_{n}\right] / J^{\prime}\right)$ where $J^{\prime}:=\mathbb{K} \otimes_{\mathbb{F}} J$.

Since $J_{n, k}$ is generated by polynomials with all coefficients equal to 1 , the generating set of $J_{n, k}$ satisfies the conditions of Lemma 3.1.

Proof. Let $\leqslant$ be any term order. It suffices to show that the quotient rings $\mathbb{F}\left[\mathbf{x}_{n}\right] / J$ and $\mathbb{K}\left[\mathbf{x}_{n}\right] / J^{\prime}$ have the same standard monomial bases with respect to $\leqslant$. To calculate the reduced Gröbner basis for the ideal $J$, we apply Buchberger's Algorithm [9, Ch. $2, \S 7$ to the generating set $\left\{f_{1}, \ldots, f_{r}\right\}$. To calculate the Gröbner basis for the ideal $J^{\prime}$, we also apply Buchberger's Algorithm to the generating set $\left\{f_{1}, \ldots, f_{r}\right\}$. In either case, all of the coefficients involved in the polynomial long division are contained in the field $\mathbb{F}$. In particular, the reduced Gröbner bases of $J$ and $J^{\prime}$ coincide. Hence, the standard monomial bases of $\mathbb{F}\left[\mathbf{x}_{n}\right] / J$ and $\mathbb{K}\left[\mathbf{x}_{n}\right] / J^{\prime}$ also coincide.

We are ready to define our point sets $Z_{n, k}$. Thanks to Lemma 3.1, we may harmlessly assume that the field $\mathbb{F}$ has at least $n+k-1$ elements by replacing $\mathbb{F}$ with an extension if necessary. We will have to choose a somewhat non-obvious point set $Z_{n, k} \subseteq \mathbb{F}^{n}$ in order to get the desired equality of ideals $\mathbf{T}\left(Z_{n, k}\right)=J_{n, k}$.

Definition 3.2. Assume $\mathbb{F}$ has at least $n+k-1$ elements and let $\alpha_{1}, \alpha_{2}, \ldots, \alpha_{n+k-1} \in$ $\mathbb{F}$ be a list of $n+k-1$ distinct field elements. Define $Z_{n, k} \subseteq \mathbb{F}^{n}$ to be the collection of points $\left(z_{1}, z_{2}, \ldots, z_{n}\right)$ such that

- for $1 \leqslant i \leqslant n$ we have $z_{i} \in\left\{\alpha_{1}, \alpha_{2}, \ldots, \alpha_{k+i-1}\right\}$,

- the coordinates $z_{1}, z_{2}, \ldots, z_{n}$ are distinct, and

- we have $\left\{\alpha_{1}, \alpha_{2}, \ldots, \alpha_{k}\right\} \subseteq\left\{z_{1}, z_{2}, \ldots, z_{n}\right\}$.

We claim that $Z_{n, k}$ is in bijective correspondence with $\mathcal{O} \mathcal{P}_{n, k}$. A bijection $\varphi$ : $\mathcal{O P}_{n, k} \rightarrow Z_{n, k}$ may be obtained as follows. Let $\sigma=\left(B_{1}|\cdots| B_{k}\right) \in \mathcal{O P}_{n, k}$ be an ordered set partition; we define $\varphi(\sigma)=\left(z_{1}, \ldots, z_{n}\right) \in Z_{n, k}$. For $1 \leqslant i \leqslant k$, we first set $z_{j}=\alpha_{i}$, where $j=\min \left(B_{i}\right)$. Write the set of unassigned indices of $\left(z_{1}, \ldots, z_{n}\right)$ as $S=[n]-\left\{\min \left(B_{1}\right), \ldots, \min \left(B_{k}\right)\right\}=\left\{s_{1}<\cdots<s_{n-k}\right\}$. For $s \in S$, let $\ell_{s}$ be the number of blocks $B$ weakly to the left of $s$ in $\sigma$ which satisfy $\min (B)<s$. Let $z_{s_{1}}=\alpha_{k+\ell_{s_{1}}}$. Assuming $z_{s_{1}}, z_{s_{2}}, \ldots, z_{s_{j-1}}$ have already been defined, let $z_{s_{j}}$ be the $\ell_{s_{j}}^{\text {th }}$ term in the sequence formed by deleting $z_{s_{1}}, z_{s_{2}}, \ldots, z_{s_{j-1}}$ from the sequence $\left(\alpha_{k+1}, \alpha_{k+2}, \ldots, \alpha_{n+k-1}\right)$.

As an example of the map $\varphi$, let $\sigma=(7|248| 13 \mid 569) \in \mathcal{O P}_{9,4}$. The following table computes the image $\varphi(\sigma)=\left(z_{1}, \ldots, z_{9}\right)$. We start by assigning the coordinates $\left(z_{7}, z_{2}, z_{1}, z_{5}\right)=\left(\alpha_{1}, \alpha_{2}, \alpha_{3}, \alpha_{4}\right)$ of the letters in the minimal blocks of $\sigma$. At the top row of the table, the coordinates corresponding to the letters $S=\{3,4,6,8,9\}$ which are not minimal in their blocks of $\sigma$ are unassigned and we have the sequence of possible values $\left(\alpha_{k+1}, \ldots, \alpha_{n+k-1}\right)=\left(\alpha_{5}, \ldots, \alpha_{12}\right)$. We add the elements of $S$ to the blocks of $\sigma$ one at a time, from smallest to largest. At each stage, we record the letter $s$ added together with the number $\ell_{s}$ of blocks $B$ weakly to the left of $s$ in $\sigma$ which satisfy $\min (B)<s$. We assign the coordinate $z_{s}$ the value of the $\ell_{s}^{t h}$ term in the list of unassigned values, and then erase the value from the list. In summary, we have

$$
\varphi:(7|248| 13 \mid 569) \mapsto\left(\alpha_{3}, \alpha_{2}, \alpha_{6}, \alpha_{5}, \alpha_{4}, \alpha_{9}, \alpha_{1}, \alpha_{8}, \alpha_{12}\right) .
$$




\begin{tabular}{|c|c|c|c|c|}
\hline$\sigma$ & $\begin{array}{l}\text { letter } s \\
\text { added }\end{array}$ & $\ell_{s}$ & unassigned $\alpha$ 's & $\varphi(\sigma)=\left(z_{1}, \ldots, z_{n}\right)$ \\
\hline$(\mathbf{7}|\mathbf{2}| \mathbf{1} \mid \mathbf{5})$ & & & $\begin{array}{c}\left(\alpha_{5}, \alpha_{6}, \alpha_{7}, \alpha_{8}, \alpha_{9}\right. \\
\left.\alpha_{10}, \alpha_{11}, \alpha_{12}\right)\end{array}$ & $\begin{array}{c}\left(\boldsymbol{\alpha}_{3}, \boldsymbol{\alpha}_{2}, z_{3}, z_{4}, \boldsymbol{\alpha}_{4}, z_{6}\right. \\
\left.\boldsymbol{\alpha}_{1}, z_{8}, z_{9}\right)\end{array}$ \\
\hline$(7|2| 13 \mid 5)$ & 3 & $\ell_{3}=2$ & $\begin{array}{c}\left(\alpha_{5}, \boldsymbol{\alpha}_{6}, \alpha_{7}, \alpha_{8}, \alpha_{9}\right. \\
\left.\alpha_{10}, \alpha_{11}, \alpha_{12}\right)\end{array}$ & $\begin{array}{c}\left(\alpha_{3}, \alpha_{2}, \boldsymbol{\alpha}_{6}, z_{4}, \alpha_{4}, z_{6}\right. \\
\left.\alpha_{1}, z_{8}, z_{9}\right)\end{array}$ \\
\hline$(7|24| 13 \mid 5)$ & 4 & $\ell_{4}=1$ & $\begin{array}{c}\left(\boldsymbol{\alpha}_{5}, \alpha_{7}, \alpha_{8}, \alpha_{9}\right. \\
\left.\alpha_{10}, \alpha_{11}, \alpha_{12}\right)\end{array}$ & $\begin{array}{c}\left(\alpha_{3}, \alpha_{2}, \alpha_{6}, \boldsymbol{\alpha}_{5}, \alpha_{4}, z_{6}\right. \\
\left.\alpha_{1}, z_{8}, z_{9}\right)\end{array}$ \\
\hline$(7|24| 13 \mid 56)$ & 6 & $\ell_{6}=3$ & $\begin{array}{c}\left(\alpha_{7}, \alpha_{8}, \boldsymbol{\alpha}_{9}, \alpha_{10}\right. \\
\left.\alpha_{11}, \alpha_{12}\right)\end{array}$ & $\begin{array}{c}\left(\alpha_{3}, \alpha_{2}, \alpha_{6}, \alpha_{5}, \alpha_{4}, \alpha_{9}\right. \\
\left.\alpha_{1}, z_{8}, z_{9}\right)\end{array}$ \\
\hline$(7|248| 13 \mid 56)$ & 8 & $\ell_{8}=2$ & $\begin{array}{c}\left(\alpha_{7}, \boldsymbol{\alpha}_{8}, \alpha_{10}, \alpha_{11},\right. \\
\left.\alpha_{12}\right)\end{array}$ & $\begin{array}{c}\left(\alpha_{3}, \alpha_{2}, \alpha_{6}, \alpha_{5}, \alpha_{4}, \alpha_{9}\right. \\
\left.\alpha_{1}, \boldsymbol{\alpha}_{8}, z_{9}\right)\end{array}$ \\
\hline$(7|248| 13 \mid 569)$ & 9 & $\ell_{9}=4$ & $\left(\alpha_{7}, \alpha_{10}, \alpha_{11}, \boldsymbol{\alpha}_{12}\right)$ & $\begin{array}{c}\left(\alpha_{3}, \alpha_{2}, \alpha_{6}, \alpha_{5}, \alpha_{4}, \alpha_{9}\right. \\
\left.\alpha_{1}, \alpha_{8}, \boldsymbol{\alpha}_{12}\right)\end{array}$ \\
\hline
\end{tabular}

We leave it for the reader to check that $\varphi: \mathcal{O} \mathcal{P}_{n, k} \rightarrow Z_{n, k}$ is well-defined and invertible. The point set $Z_{n, k}$ therefore achieves Step 1 of our strategy.

Achieving Step 2 of our strategy involves showing that the generators of $J_{n, k}$ arise as top degree components of strategically chosen polynomials vanishing on $Z_{n, k}$.

Lemma 3.3. Assume $\mathbb{F}$ has at least $n+k-1$ elements. We have $J_{n, k} \subseteq \mathbf{T}\left(Z_{n, k}\right)$.

Proof. It suffices to show that every generator of $J_{n, k}$ arises as the top degree component of a polynomial in $\mathbf{I}\left(Z_{n, k}\right)$. Let us first consider the generators $h_{k}\left(x_{1}\right), h_{k}\left(x_{1}, x_{2}\right), \ldots, h_{k}\left(x_{1}, x_{2}, \ldots, x_{n}\right)$.

For $1 \leqslant i \leqslant n$, we claim that

$$
\sum_{j \geqslant 0}(-1)^{j} h_{k-j}\left(x_{1}, x_{2}, \ldots, x_{i}\right) e_{j}\left(\alpha_{1}, \alpha_{2}, \ldots, \alpha_{k+i-1}\right) \in \mathbf{I}\left(Z_{n, k}\right) .
$$

Indeed, this alternating sum is the coefficient of $t^{k}$ in the power series expansion of the rational function

$$
\frac{\left(1-\alpha_{1} t\right)\left(1-\alpha_{2} t\right) \cdots\left(1-\alpha_{k+i-1} t\right)}{\left(1-x_{1} t\right)\left(1-x_{2} t\right) \cdots\left(1-x_{i} t\right)} .
$$

If $\left(x_{1}, \ldots, x_{n}\right) \in Z_{n, k}$, by the definition of $Z_{n, k}$ the terms in the denominator cancel with $i$ terms in the numerator, yielding a polynomial in $t$ of degree $k-1$. The assertion (56) follows. Taking the highest degree component, we get $h_{k}\left(x_{1}, x_{2}, \ldots, x_{i}\right) \in$ $\mathbf{T}\left(Z_{n, k}\right)$.

Next, we show $e_{r}\left(x_{1}, \ldots, x_{n}\right) \in \mathbf{T}\left(Z_{n, k}\right)$ for $n-k<r \leqslant n$. To prove this, we claim that

$$
\sum_{j \geqslant 0}(-1)^{j} e_{r-j}\left(x_{1}, \ldots, x_{n}\right) h_{j}\left(\alpha_{1}, \ldots, \alpha_{n+k-1}\right) \in \mathbf{I}\left(Z_{n, k}\right) .
$$

Indeed, this alternating sum is the coefficient of $t^{r}$ in the rational function

$$
\frac{\left(1+x_{1} t\right)\left(1+x_{2} t\right) \cdots\left(1+x_{n} t\right)}{\left(1+\alpha_{1} t\right)\left(1+\alpha_{2} t\right) \cdots\left(1+\alpha_{k} t\right)} .
$$

If $\left(x_{1}, \ldots, x_{n}\right) \in Z_{n, k}$, the terms in the denominator cancel with $k$ terms in the numerator, yielding a polynomial in $t$ of degree $n-k$. Since $r>n-k$, the assertion (58) follows. Taking the highest degree component, we get $e_{r}\left(x_{1}, \ldots, x_{n}\right) \in \mathbf{T}\left(Z_{n, k}\right)$. 
3.2. The Hilbert Series of $S_{n, k}$. Let $<$ be the neglex term order on $\mathbb{F}\left[\mathbf{x}_{n}\right]$. We are ready to execute Step 3 of our strategy and describe the standard monomial basis of the quotient $S_{n, k}$. To do so, we recall the definition of 'skip monomials' in $\mathbb{F}\left[\mathbf{x}_{n}\right]$ of [16].

Let $S=\left\{s_{1}<\cdots<s_{m}\right\} \subseteq[n]$ be a set. Following [16, Defn. 3.2], the skip monomial $\mathbf{x}(S)$ is the monomial in $\mathbb{F}\left[\mathbf{x}_{n}\right]$ given by

$$
\mathbf{x}(S):=x_{s_{1}}^{s_{1}} x_{s_{2}}^{s_{2}-1} \cdots x_{s_{m}}^{s_{m}-m+1} .
$$

For example, we have $\mathbf{x}(2578)=x_{2}^{2} x_{5}^{4} x_{7}^{5} x_{8}^{5}$. The adjective 'skip' refers to the fact that the exponent sequence $\mathbf{x}(S)$ increases whenever the set $S$ skips a letter. Our variable order convention will require us to consider the reverse skip monomial

$$
\mathbf{x}(S)^{*}:=x_{n-s_{1}+1}^{s_{1}} x_{n-s_{2}+1}^{s_{2}-1} \cdots x_{n-s_{m}+1}^{s_{m}-m+1} .
$$

For example, if $n=9$ we have $\mathbf{x}(2578)^{*}=x_{8}^{2} x_{5}^{4} x_{3}^{5} x_{2}^{5}$. The following definition is the reverse of [16, Defn. 4.4].

Definition 3.4. Let $k \leqslant n$ be positive integers. A monomial $m \in \mathbb{F}\left[\mathbf{x}_{n}\right]$ is $(n, k)$ reverse nonskip if

- $x_{i}^{k} \nmid m$ for $1 \leqslant i \leqslant n$, and

- $\mathbf{x}(S)^{*} \nmid m$ for all $S \subseteq[n]$ with $|S|=n+k-1$.

Let $\mathcal{C}_{n, k}$ denote the collection of all $(n, k)$-reverse nonskip monomials in $\mathbb{F}\left[\mathbf{x}_{n}\right]$.

There is some redundancy in Definition 3.4. In particular, if $n \in S$, the power of $x_{1}$ in $\mathbf{x}(S)^{*}$ where $|S|=n-k+1$ is $x_{1}^{k}$, so that we need only consider those sets $S$ with $n \notin S$.

Theorem 3.5. Let $\mathbb{F}$ be any field and $\leqslant$ be the neglex term order on $\mathbb{F}\left[\mathbf{x}_{n}\right]$. The standard monomial basis of $S_{n, k}=\mathbb{F}\left[\mathbf{x}_{n}\right] / J_{n, k}$ with respect to $\leqslant$ is $\mathcal{C}_{n, k}$.

Proof. By the definition of neglex, we have

$$
\operatorname{in}_{<}\left(h_{k}\left(x_{1}, x_{2}, \ldots, x_{i}\right)\right)=x_{i}^{k} \in \operatorname{in}_{<}\left(J_{n, k}\right) .
$$

By $\left[16\right.$, Lem. 3.4, Lem. 3.5] we also have $\mathbf{x}(S)^{*} \in \operatorname{in}_{<}\left(J_{n, k}\right)$ whenever $S \subseteq[n]$ satisfies $|S|=n-k+1$. It follows that $\mathcal{C}_{n, k}$ contains the standard monomial basis of $S_{n, k}$.

To prove that $\mathcal{C}_{n, k}$ is the standard monomial basis of $S_{n, k}$, it suffices to show $\left|\mathcal{C}_{n, k}\right| \leqslant \operatorname{dim}\left(S_{n, k}\right)$. Thanks to Lemma 3.1 , we may replace $\mathbb{F}$ by an extension if necessary to assume that $\mathbb{F}$ contains at least $n+k-1$ elements. By Lemma 3.3, we have

(63) $\operatorname{dim}\left(S_{n, k}\right)=\operatorname{dim}\left(\mathbb{F}\left[\mathbf{x}_{n}\right] / J_{n, k}\right) \geqslant \operatorname{dim}\left(\mathbb{F}\left[\mathbf{x}_{n}\right] / \mathbf{T}\left(Z_{n, k}\right)\right)=\left|Z_{n, k}\right|=\left|\mathcal{O} \mathcal{P}_{n, k}\right|$.

On the other hand, [16, Thm. 4.9] implies (after reversing variables) that $\left|\mathcal{O} \mathcal{P}_{n, k}\right|=$ $\left|\mathcal{C}_{n, k}\right|$.

When $k=n$, the collection $\mathcal{C}_{n, n}$ consists of sub-staircase monomials $x_{1}^{a_{1}} \cdots x_{n}^{a_{n}}$ whose exponent sequences satisfy $0 \leqslant a_{i} \leqslant n-i$; this is the basis for the coinvariant algebra obtained by E. Artin [2] using Galois theory. Let us mention an analogous characterization of $\mathcal{C}_{n, k}$ which was derived in [16].

Recall that a shuffle of two sequences $\left(a_{1}, \ldots, a_{r}\right)$ and $\left(b_{1}, \ldots, b_{s}\right)$ is an interleaving $\left(c_{1}, \ldots, c_{r+s}\right)$ of these sequences which preserves the relative order of the $a$ 's and the $b$ 's. A $(n, k)$-staircase is a shuffle of the sequences $(k-1, k-2, \ldots, 1,0)$ and $(k-1, k-1, \ldots, k-1)$, where the second sequence has $n-k$ copies of $k-1$. For example, the $(5,3)$-staircases are the shuffles of $(2,1,0)$ and $(2,2)$ :

$(2,1,0,2,2),(2,1,2,0,2),(2,2,1,0,2),(2,1,2,2,0),(2,2,1,2,0)$, and $(2,2,2,1,0)$. The following theorem is just the reversal of [16, Thm. 4.13]. 
Corollary 3.6 ([16, Thm. 4.13]). The monomial basis $\mathcal{C}_{n, k}$ of $S_{n, k}$ is the set of monomials $x_{1}^{a_{1}} x_{2}^{a_{2}} \cdots x_{n}^{a_{n}}$ in $\mathbb{F}\left[\mathbf{x}_{n}\right]$ whose exponent sequences $\left(a_{1}, a_{2}, \ldots, a_{n}\right)$ are componentwise $\leqslant$ some $(n, k)$-staircase.

For example, consider the case $(n, k)=(4,2)$. The $(4,2)$-staircases are the shuffles of $(1,0)$ and $(1,1)$ :

It follows that

$$
(1,0,1,1),(1,1,0,1) \text {, and }(1,1,1,0)
$$

$$
\mathcal{C}_{4,2}=\left\{1, x_{1}, x_{2}, x_{3}, x_{4}, x_{1} x_{2}, x_{1} x_{3}, x_{1} x_{4}, x_{2} x_{3}, x_{2} x_{4}, x_{3} x_{4}, x_{1} x_{3} x_{4}, x_{1} x_{2} x_{4}, x_{1} x_{2} x_{3}\right\}
$$

is the standard monomial basis of $S_{4,2}$ with respect to neglex. Consequently, we have the Hilbert series

$$
\operatorname{Hilb}\left(S_{4,2} ; q\right)=1+4 q+6 q^{2}+3 q^{3} \text {. }
$$

We can also describe a Gröbner basis of the ideal $J_{n, k}$. For $\gamma=\left(\gamma_{1}, \ldots, \gamma_{n}\right)$ a weak composition (i.e., possibly containing 0's) of length $n$, let $\kappa_{\gamma}\left(\mathbf{x}_{n}\right) \in \mathbb{F}\left[\mathbf{x}_{n}\right]$ be the associated Demazure character (see e.g. [16, Sec. 2.4]).

If $S \subseteq[n]$, let $\gamma(S)=\left(\gamma_{1}, \ldots, \gamma_{n}\right)$ be the exponent sequence of the corresponding skip monomial $\mathbf{x}(S)$. That is, if $S=\left\{s_{1}<\cdots<s_{m}\right\}$ we have

$$
\gamma_{i}= \begin{cases}s_{j}-j+1 & \text { if } i=s_{j} \in S \\ 0 & \text { if } i \notin S .\end{cases}
$$

Let $\gamma(S)^{*}=\left(\gamma_{n}, \ldots, \gamma_{1}\right)$ be the reverse of the weak composition $\gamma(S)$. In particular, we can consider the Demazure character $\kappa_{\gamma(S)^{*}}\left(\mathbf{x}_{n}\right) \in \mathbb{F}\left[\mathbf{x}_{n}\right]$.

THEOREM 3.7. Let $k \leqslant n$ be positive integers and let $\leqslant$ be the neglex term order on $\mathbb{F}\left[\mathbf{x}_{n}\right]$. The polynomials

$$
h_{k}\left(x_{1}\right), h_{k}\left(x_{1}, x_{2}\right), \ldots, h_{k}\left(x_{1}, x_{2}, \ldots, x_{n}\right)
$$

together with the Demazure characters

$$
\kappa_{\gamma(S) *}\left(\mathbf{x}_{n}\right) \in \mathbb{F}\left[\mathbf{x}_{n}\right]
$$

for all $S \subseteq[n-1]$ satisfying $|S|=n-k+1$, form a Gröbner basis for the ideal $J_{n, k}$.

When $k<n$, this Gröbner basis is minimal.

For example, if $(n, k)=(6,4)$, a Gröbner basis of $J_{6,4} \subseteq \mathbb{F}\left[\mathbf{x}_{6}\right]$ is given by the polynomials

$$
\begin{gathered}
h_{4}\left(x_{1}\right), \quad h_{4}\left(x_{1}, x_{2}\right), \quad h_{4}\left(x_{1}, x_{2}, x_{3}\right), \quad h_{4}\left(x_{1}, x_{2}, x_{3}, x_{4}\right), \\
h_{4}\left(x_{1}, x_{2}, x_{3}, x_{4}, x_{5}\right) \quad \text { and } \quad h_{4}\left(x_{1}, x_{2}, x_{3}, x_{4}, x_{5}, x_{6}\right)
\end{gathered}
$$

together with the Demazure characters

$$
\begin{aligned}
& \kappa_{(0,0,0,1,1,1)}\left(\mathbf{x}_{6}\right), \kappa_{(0,0,2,0,1,1)}\left(\mathbf{x}_{6}\right), \kappa_{(0,3,0,0,1,1)}\left(\mathbf{x}_{6}\right), \kappa_{(0,0,2,2,0,1)}\left(\mathbf{x}_{6}\right), \kappa_{(0,3,0,2,0,1)}\left(\mathbf{x}_{6}\right), \\
& \kappa_{(0,3,3,0,0,1)}\left(\mathbf{x}_{6}\right), \kappa_{(0,0,2,2,2,0)}\left(\mathbf{x}_{6}\right), \kappa_{(0,3,0,2,2,0)}\left(\mathbf{x}_{6}\right), \kappa_{(0,3,3,0,2,0)}\left(\mathbf{x}_{6}\right), \kappa_{(0,3,3,3,0,0)}\left(\mathbf{x}_{6}\right) .
\end{aligned}
$$

Proof. We need to show that the polynomials in question lie in the ideal $J_{n, k}$. This is clear for the polynomials $h_{k}\left(x_{1}, \ldots, x_{i}\right)$. For the Demazure characters, we apply [16, Lem. 3.4] (and in particular [16, Eqn. 3.4]) to see that $\kappa_{\gamma(S)^{*}}\left(\mathbf{x}_{n}\right) \in J_{n, k}$ whenever $S \subseteq[n-1]$ satisfies $|S|=n-k+1$.

Next we examine the leading terms of the polynomials in question. It is evident that

$$
\operatorname{in}_{<}\left(h_{k}\left(x_{1}, \ldots, x_{i}\right)\right)=x_{i}^{k}
$$

After applying variable reversal to [16, Lem. 3.5], we see that

$$
\operatorname{in}_{<}\left(\kappa_{\gamma(S)^{*}}\left(\mathbf{x}_{n}\right)\right)=\mathbf{x}(S)^{*} .
$$


By Theorem 3.5 and the remarks following Definition 3.4, it follows that these monomials generate the initial ideal in $\operatorname{in}_{<}\left(J_{n, k}\right)$ of $J_{n, k}$.

When $k<n$, observe that for $S \subseteq[n-1]$ with $|S|=n-k+1$, the monomial $\mathbf{x}(S)^{*}$ has support $\{i: n-i+1 \in S\}$. Moreover, the monomial $\mathbf{x}(S)^{*}$ does not contain any exponents $\geqslant k$ since $n \notin S$. The minimality of the Gröbner basis follows.

Theorem 3.7 is the 0 -Hecke analog of [16, Thm. 4.14]. Unlike the case of [16, Thm. 4.14], the Gröbner basis of Theorem 3.7 is not reduced. When $k=n$, the ideal $J_{n, n}$ is the classical invariant ideal $I_{n}$ and has reduced Gröbner basis $\left\{h_{1}\left(x_{1}, \ldots, x_{n}\right), h_{2}\left(x_{1}, \ldots, x_{n-1}\right), \ldots, h_{n}\left(x_{1}\right)\right\}$. The authors do not have a conjecture for the reduced Gröbner basis for the ideal $J_{n, k}$. The work of [16] gives us a formula for the Hilbert series of $S_{n, k}$.

THEOREM 3.8. Let $k \leqslant n$ be positive integers. We have $\operatorname{Hilb}\left(S_{n, k} ; q\right)=\operatorname{rev}_{q}\left([k] !_{q}\right.$. $\left.\operatorname{Stir}_{q}(n, k)\right)$.

Proof. By Theorem 3.5 and [16, Thm. 4.13], the Hilbert series of $S_{n, k}$ equals the Hilbert series of $R_{n, k}$. Applying [16, Thm. 4.10] finishes the proof.

\section{Garsia-Stanton type bases}

Let $k \leqslant n$ be positive integers. Given a composition $\alpha \models n$ and a length $n$ sequence $\mathbf{i}=\left(i_{1}, \ldots, i_{n}\right)$ of nonnegative integers, define a monomial $\mathbf{x}_{\alpha, \mathbf{i}} \in \mathbb{F}\left[\mathbf{x}_{n}\right]$ by

$$
\mathbf{x}_{\alpha, \mathbf{i}}:=\left(\prod_{j \in \operatorname{Des}(\alpha)} x_{1} x_{2} \cdots x_{j}\right) x_{1}^{i_{1}} x_{2}^{i_{2}} \cdots x_{n}^{i_{n}} .
$$

If $w \in \mathfrak{S}_{n}$ is a permutation and $\mathbf{i}=\left(i_{1}, \ldots, i_{n}\right)$ is a sequence of nonnegative integers, we define the generalized Garsia-Stanton monomial $g s_{w, \mathbf{i}}:=w\left(\mathbf{x}_{\alpha, \mathbf{i}}\right)$, where $\alpha \models n$ is characterized by $\operatorname{Des}(\alpha)=\operatorname{Des}(w)$. The degree of $g s_{w, \mathbf{i}}$ is given by $\operatorname{deg}\left(g s_{w, \mathbf{i}}\right)=$ $\operatorname{maj}(w)+|\mathbf{i}|$, where $|\mathbf{i}|:=i_{1}+\cdots+i_{n}$.

For example, let $(n, k)=(9,5), w=254689137 \in \mathfrak{S}_{9}$ and $\mathbf{i}=(2,2,1,1,0,0,0,0,0)$. We have $\operatorname{Des}(w)=\{2,6\}$, so that the composition $\alpha \models 9$ with $\operatorname{Des}(\alpha)=\operatorname{Des}(w)$ is $\alpha=(2,4,3)$. It follows that

$$
\mathbf{x}_{\alpha, \mathbf{i}}=\left(x_{1} x_{2}\right)\left(x_{1} x_{2} x_{3} x_{4} x_{5} x_{6}\right)\left(x_{1}^{2} x_{2}^{2} x_{3}^{1} x_{4}^{1}\right) .
$$

The corresponding generalized GS monomial is

$$
g s_{w, \mathbf{i}}=\left(x_{2} x_{5}\right)\left(x_{2} x_{5} x_{4} x_{6} x_{8} x_{9}\right)\left(x_{2}^{2} x_{5}^{2} x_{4}^{1} x_{6}^{1}\right) .
$$

Haglund, Rhoades, and Shimozono introduced [16, Defn. 5.2] (using different notation) the following collection $\mathcal{G S}_{n, k}$ of monomials:

$$
\mathcal{G S}_{n, k}:=\left\{g s_{w, \mathbf{i}}: w \in \mathfrak{S}_{n}, k-\operatorname{des}(w)>i_{1} \geqslant \cdots \geqslant i_{n-k} \geqslant 0=i_{n-k+1}=\cdots=i_{n}\right\} .
$$

When $k=n$, we have $g s_{w, \mathbf{i}} \in \mathcal{G S}_{n, n}$ if and only if $w \in \mathfrak{S}_{n}$ and $\mathbf{i}=0^{n}$ is the sequence of $n$ zeros. Garsia [10] proved that $\mathcal{G S}_{n, n}$ descends to a basis of the classical coinvariant algebra $R_{n}$. Garsia and Stanton [12] later studied $\mathcal{G S}_{n, n}$ in the context of Stanley-Reisner theory. Extending Garsia's result, Haglund et. al. proved that $\mathcal{G S}_{n, k}$ descends to a basis of $R_{n, k}$ [16, Thm. 5.3]. We will prove that $\mathcal{G S}_{n, k}$ also descends to a basis of $S_{n, k}$. In fact, we will prove that $\mathcal{G S}_{n, k}$ is just one of a family of bases of $S_{n, k}$.

Huang used isobaric Demazure operators to define a basis of the classical coinvariant algebra $R_{n}$ which is related to the classical GS basis $\mathcal{G S}_{n, n}$ by a unitriangular transition matrix [17]. We will modify $\mathcal{G S}_{n, k}$ to get a new basis of $S_{n, k}$ in an analogous way. As in [17], our modified basis will be crucial in our analysis of the $H_{n}(0)$-module 
structure of $S_{n, k}$. This modified basis and $\mathcal{G S}_{n, k}$ itself will both belong to the following family of bases of $S_{n, k}$.

To describe these bases, we will need a partial order on monomials in $\mathbb{F}\left[\mathbf{x}_{n}\right]$. If $m=x_{1}^{a_{1}} \cdots x_{n}^{a_{n}}$ is a monomial in $\mathbb{F}\left[\mathbf{x}_{n}\right]$, let $\lambda(m):=\operatorname{sort}\left(a_{1}, \ldots, a_{n}\right)$ be the sequence obtained by sorting the exponent sequence of $m$ into weakly decreasing order. Following Adin, Brenti, and Roichman [1], we associate a collection of objects to any monomial $m=x_{1}^{a_{1}} \cdots x_{n}^{a_{n}}$ in $\mathbb{F}\left[\mathbf{x}_{n}\right]$ as follows. Let $\sigma(m)=\sigma_{1} \cdots \sigma_{n} \in \mathfrak{S}_{n}$ be the permutation (in one-line notation) obtained by listing the indices of variables in weakly decreasing order of the exponents in $m$, breaking ties by listing smaller indexed variables first. Let $d(m)=\left(d_{1}, \ldots, d_{n}\right)$ be the integer sequence given by $d_{j}=|\operatorname{Des}(\sigma(m)) \cap\{j, j+1, \ldots, n\}|$. Adin, Brenti, and Roichman [1] showed that the componentwise difference $\lambda(m)-d(m)$ is an integer partition (i.e., has weakly decreasing components). Let $\mu(m)$ be the conjugate of this integer partition.

For example, if $m=x_{1}^{3} x_{2}^{4} x_{3}^{0} x_{4}^{2} x_{5}^{2} x_{6}^{0} x_{7}^{0}$, then $\lambda(m)=(4,3,2,2,0,0,0)$ and $\sigma(m)=$ 2145367. It follows that $d(m)=(2,1,1,1,0,0,0), \lambda(m)-d(m)=(2,2,1,1,0,0,0)$, and $\mu(m)=(4,2)$.

DEFINITION 4.1. Let $\prec$ be the partial order on monomials in $\mathbb{F}\left[\mathbf{x}_{n}\right]$ defined by $m \prec m^{\prime}$ if and only if $\lambda(m)<\lambda\left(m^{\prime}\right)$ in lexicographical order.

LEMMA 4.2. Let $\mathcal{B}_{n, k}=\left\{b_{w, \mathbf{i}}\right\}$ be a set of polynomials indexed by pairs $(w, \mathbf{i})$ where $w \in \mathfrak{S}_{n}$ and $\mathbf{i}=\left(i_{1}, \ldots, i_{n}\right)$ satisfy

$$
k-\operatorname{des}(w)>i_{1} \geqslant \cdots \geqslant i_{n-k} \geqslant 0=i_{n-k+1}=\cdots=i_{n} .
$$

Assume that any $b_{w, \mathbf{i}} \in \mathcal{B}_{n, k}$ has the form

$$
b_{w, \mathbf{i}}=g s_{w, \mathbf{i}}+\sum_{m \prec g s_{w, \mathbf{i}}} c_{m} \cdot m
$$

where the $c_{m} \in \mathbb{F}$ are scalars which could depend on $(w, \mathbf{i})$ and $\prec$ is the partial order on monomials appearing in Definition 4.1. The set $\mathcal{B}_{n, k}$ descends to a basis of $S_{n, k}$.

Proof. By [16, Thm. 5.3], we know that $\left|\mathcal{B}_{n, k}\right|=\left|\mathcal{G S}_{n, k}\right|=\left|\mathcal{O} \mathcal{P}_{n, k}\right|$. By Theorem 3.8, we have $\operatorname{dim}\left(S_{n, k}\right)=\left|\mathcal{O} \mathcal{P}_{n, k}\right|$. Therefore, it is enough to show that $\mathcal{B}_{n, k}$ descends to a spanning set of $S_{n, k}$.

If $\mathcal{B}_{n, k}$ did not descend to a spanning set of $S_{n, k}$, then there would be a monomial $m \in \mathbb{F}\left[\mathbf{x}_{n}\right]$ whose image $m+J_{n, k}$ did not lie in the span of $\mathcal{B}_{n, k}$. Working towards a contradiction, suppose that such a monomial existed.

Let $m=x_{1}^{a_{1}} \cdots x_{n}^{a_{n}}$ be any monomial in $\mathbb{F}\left[\mathbf{x}_{n}\right]$. We argue that $m$ is expressible modulo $J_{n, k}$ as a linear combination of monomials of the form $m^{\prime}=x_{1}^{b_{1}} \cdots x_{n}^{b_{n}}$ with $b_{i}<k$ for all $i$. Indeed, if $m$ does not already have this form, choose $i$ maximal such that $a_{i}>k$. Since $h_{k}\left(x_{1}, \ldots, x_{i}\right) \in J_{n, k}$, modulo $J_{n, k}$ we have

$$
m \equiv-\left(x_{1}^{a_{1}} \cdots x_{i}^{a_{i}-k} \cdots x_{n}^{a_{n}}\right) \sum_{\substack{1 \leqslant j_{1} \leqslant \cdots \leqslant j_{k} \leqslant i \\ j_{1} \neq i}} x_{j_{1}} \cdots x_{j_{k}} .
$$

If every monomial appearing on the right hand side is of the required form, we are done. Otherwise, we may iterate this procedure. Since $h_{k}\left(x_{1}\right)=x_{1}^{k} \in J_{n, k}$, iterating this procedure eventually yields 0 or a linear combination of monomials of the required form.

Let $\prec_{A B R}$ be the partial order on monomials in $\mathbb{F}\left[\mathbf{x}_{n}\right]$ defined by $m \prec_{A B R} m^{\prime}$ if and only if $\lambda(m)<\lambda\left(m^{\prime}\right)$ in lexicographical order or $\left(\lambda(m)=\lambda\left(m^{\prime}\right)\right.$ and $\operatorname{inv}(\sigma(m))>$ $\left.\operatorname{inv}\left(\sigma\left(m^{\prime}\right)\right)\right)$. In particular, the relation $m \prec m^{\prime}$ implies $m \prec_{A B R} m^{\prime}$. 
Let $m=x_{1}^{a_{1}} \cdots x_{n}^{a_{n}}$ be any monomial in $\mathbb{F}\left[\mathbf{x}_{n}\right]$ such that $m+J_{n, k}$ does not lie in the span of $\mathcal{B}_{n, k}$. By the reasoning above, we may assume that $a_{i}<k$ for all $1 \leqslant i \leqslant n$. Choose such an $m$ which is minimal with respect to the partial order $\prec_{A B R}$.

Adin, Brenti, and Roichman [1, Lem. 3.5] proved that we can 'straighten' the monomial $m$ and write

$$
m=g s_{\sigma(m)} e_{\mu(m)}\left(\mathbf{x}_{n}\right)-\Sigma,
$$

where $\Sigma$ is a linear combination of monomials which are $\prec_{A B R} m$. Here

$$
g s_{\sigma(m)}:=g s_{\sigma(m), 0^{n}}=x_{\sigma_{1}}^{d_{1}} \cdots x_{\sigma_{n}}^{d_{n}}
$$

is the 'classical' GS monomial indexed by $\sigma(m)$. Our assumption on $m$ guarantees that $\Sigma$ lies in the span of $\mathcal{B}_{n, k}$ modulo $J_{n, k}$.

If $\mu(m)_{1}>n-k$, then $e_{\mu(m)}\left(\mathbf{x}_{n}\right) \equiv 0$ modulo $J_{n, k}$. It follows that $m$ lies in the span of $\mathcal{B}_{n, k}$ modulo $J_{n, k}$, which is a contradiction.

If $\mu(m)_{1} \leqslant n-k$, then by the definition of $\lambda(m), d(m)$, and $\mu(m)$, we may write

$$
m=g s_{\sigma(m)} \cdot x_{\sigma_{1}}^{\mu(m)_{1}^{\prime}} \cdots x_{\sigma_{n-k}}^{\mu(m)_{n-k}^{\prime}},
$$

where $\mu(m)_{1}^{\prime} \geqslant \cdots \geqslant \mu(m)_{n-k}^{\prime} \geqslant 0$ is the conjugate of $\mu(m)$. Suppose $\mu(m)_{1}^{\prime} \geqslant$ $k-\operatorname{des}(\sigma(m))$. Since the exponent of $x_{\sigma_{1}}$ in $g s_{\sigma(m)}$ equals $\operatorname{des}(\sigma(m))$, we then have $x_{\sigma_{1}}^{k} \mid m$, which contradicts the assumption that $m$ has no variables with power $\geqslant k$. Therefore, we have $\mu(m)_{1}^{\prime}<k-\operatorname{des}(\sigma(m))$. This means that $m \in \mathcal{G S}_{n, k}$ and $m=$ $g s_{w, \mathbf{i}}$ for some pair $(w, \mathbf{i})$. (In fact, we can take $(w, \mathbf{i})=\left(\sigma(m), \mu^{\prime}\right)$.) However, our assumption on $\mathcal{B}_{n, k}$ guarantees that

$$
m=g s_{w, \mathbf{i}}=b_{w, \mathbf{i}}-\sum_{m^{\prime} \prec m} c_{m^{\prime}} \cdot m^{\prime}
$$

for some scalars $c_{m^{\prime}} \in \mathbb{F}$. Then our assumption on $m$ together with the fact $\left(m^{\prime} \prec\right.$ $\left.m \Rightarrow m^{\prime} \prec_{A B R} m\right)$ imply that $m$ lies in the span of $\mathcal{B}_{n, k}$ modulo $J_{n, k}$, which is a contradiction.

Corollary 4.3. Let $k \leqslant n$ be positive integers. The set $\mathcal{G S}_{n, k}$ of generalized GarsiaStanton monomials descends to a basis of $S_{n, k}$.

For example, suppose $(n, k)=(7,5)$ and $w=6123745$. Then $\operatorname{des}(w)=2$ and the classical GS monomial is $g s_{w}=\left(x_{6}\right)\left(x_{6} x_{1} x_{2} x_{3} x_{7}\right)$. We have $n-k=2$ and $k-\operatorname{des}(w)=3$, so that this classical GS monomial gives rise to the following six elements of $\mathcal{G S}_{n, k}$ :

$$
\begin{array}{ccc}
\left(x_{6}\right)\left(x_{6} x_{1} x_{2} x_{3} x_{7}\right) & \left(x_{6}\right)\left(x_{6} x_{1} x_{2} x_{3} x_{7}\right)\left(x_{6}\right) & \left(x_{6}\right)\left(x_{6} x_{1} x_{2} x_{3} x_{7}\right)\left(x_{6}^{2}\right) \\
\left(x_{6}\right)\left(x_{6} x_{1} x_{2} x_{3} x_{7}\right)\left(x_{6} x_{2}\right) & \left(x_{6}\right)\left(x_{6} x_{1} x_{2} x_{3} x_{7}\right)\left(x_{6}^{2} x_{2}\right) & \left(x_{6}\right)\left(x_{6} x_{1} x_{2} x_{3} x_{7}\right)\left(x_{6}^{2} x_{2}^{2}\right) .
\end{array}
$$

\section{Module structure over the 0-Hecke Algebra}

In this section we prove an isomorphism $S_{n, k} \cong \mathbb{F}\left[\mathcal{O P}{ }_{n, k}\right]$ of (ungraded) $H_{n}(0)$ modules.

5.1. ORderEd SET PARTITIONS. We first describe the $H_{n}(0)$-module structure of $\mathbb{F}\left[\mathcal{O P}{ }_{n, k}\right]$. Recall that if $\alpha \models n$ is a composition, then $P_{\alpha}$ is the corresponding indecomposable projective $H_{n}(0)$-module. We need a family of projective $H_{n}(0)$-modules which are indexed by pairs of compositions related by refinement. Let $\alpha, \beta \models n$ be two compositions satisfying $\alpha \preceq \beta$. Let $P_{\alpha, \beta}$ be the $H_{n}(0)$-module given by

$$
P_{\alpha, \beta}:=H_{n}(0) \bar{\pi}_{w_{0}(\alpha)} \pi_{w_{0}\left(\beta^{c}\right)} .
$$

In particular, we have $P_{\alpha, \alpha}=P_{\alpha}$. More generally, we have the following structural result on $P_{\alpha, \beta}$. 
Lemma 5.1 (Huang [18, Thm. 3.2]). Let $\alpha, \beta \models n$ and assume $\alpha \preceq \beta$. Then $P_{\alpha, \beta}$ has basis

$$
\left\{\bar{\pi}_{w} \pi_{w_{0}\left(\beta^{c}\right)}: w \in \mathfrak{S}_{n}, \operatorname{Des}(\alpha) \subseteq \operatorname{Des}(w) \subseteq \operatorname{Des}(\beta)\right\}
$$

and direct sum decomposition

$$
P_{\alpha, \beta} \cong \bigoplus_{\alpha \preceq \gamma \preceq \beta} P_{\gamma} .
$$

For example, the module $P_{(4,1),(1,2,1,1)}$ breaks up into projective indecomposable submodules as

$$
P_{(4,1),(1,2,1,1)} \cong P_{(4,1)} \oplus P_{(1,3,1)} \oplus P_{(3,1,1)} \oplus P_{(1,2,1,1)} .
$$

Recall that, for each composition $\alpha=\left(\alpha_{1}, \ldots, \alpha_{\ell}\right) \models n$, we denote by $\mathcal{O} \mathcal{P}_{\alpha}$ the collection of ordered set partitions of shape $\alpha$, i.e., pairs $(w, \alpha)$ for all $w \in \mathfrak{S}_{n}$ with $\operatorname{Des}(w) \subseteq \operatorname{Des}(\alpha)$.

Lemma 5.2. Let $\alpha=\left(\alpha_{1}, \ldots, \alpha_{\ell}\right)$ be a composition of $n$. Then $\mathbb{F}\left[\mathcal{O P}_{\alpha}\right]$ is a cyclic $H_{n}(0)$-module generated by the ordered set partition $(12 \cdots n, \alpha)$ and is isomorphic to $P_{(n), \alpha}$ via the map defined by sending $(w, \alpha)$ to $\bar{\pi}_{w} \pi_{w_{0}\left(\alpha^{c}\right)}$ for all $w \in \mathfrak{S}_{n}$ with $\operatorname{Des}(w) \subseteq \operatorname{Des}(\alpha)$.

Proof. Huang $[18,(3.3)]$ defined an action of $H_{n}(0)$ on the $\mathbb{F}$-span $P_{\alpha_{1} \oplus \cdots \oplus \alpha_{\ell}}$ of standard tableaux of skew shape $\alpha_{1} \oplus \cdots \oplus \alpha_{\ell}$, where $\alpha_{1} \oplus \cdots \oplus \alpha_{\ell}$ is a disconnected union of rows of lengths $\alpha_{1}, \ldots, \alpha_{\ell}$, ordered from southwest to northeast. There is an obvious isomorphism $\mathbb{F}\left[\mathcal{O P}_{\alpha}\right] \cong P_{\alpha_{1} \oplus \cdots \oplus \alpha_{\ell}}$ by sending an ordered set partition $\left(B_{1}|\cdots| B_{k}\right)$ to the tableau whose rows are $B_{1}, \ldots, B_{k}$ from southwest to northeast. Combining this with the isomorphism $P_{\alpha_{1} \oplus \cdots \oplus \alpha_{\ell}} \cong P_{(n), \alpha}$ provided by [18, Thm. 3.3] gives the desired result.

Proposition 5.3. Let $k \leqslant n$ be positive integers. Then we have isomorphisms of $H_{n}(0)$-modules:

$$
\mathbb{F}\left[\mathcal{O P}{ }_{n, k}\right] \cong \bigoplus_{\substack{\alpha \models n \\
\ell(\alpha)=k}} \mathbb{F}\left[\mathcal{O P} \mathcal{P}_{\alpha}\right] \cong \bigoplus_{\beta \models n} P_{\beta}^{\oplus\left(\begin{array}{c}
n-\ell(\beta) \\
k-\ell(\beta)
\end{array}\right)} .
$$

Proof. Since $\mathcal{O P}_{n, k}$ is the disjoint union of $\mathcal{O P}$, for all compositions $\alpha \models n$ of length $\ell(\alpha)=k$, the first desired isomorphism follows. Applying Lemma 5.1 and Lemma 5.2 to each $\mathcal{O P} \mathcal{P}_{\alpha}$ gives a direct sum decomposition of $\mathbb{F}\left[\mathcal{O P}_{n, k}\right]$ into projective indecomposable modules. The multiplicity of $P_{\beta}$ in this direct sum equals

$$
|\{\beta \preceq \alpha: \ell(\alpha)=k\}|=\left(\begin{array}{l}
n-\ell(\beta) \\
k-\ell(\beta)
\end{array}\right)
$$

for each $\beta \models n$. The second desired isomorphism follows.

For example, when $n=4$ and $k=2$ we have $\mathbb{F}[\mathcal{O P}(1,3)] \cong P_{(1,3)} \oplus P_{(4)}, \mathbb{F}[\mathcal{O P}(2,2)] \cong$ $P_{(2,2)} \oplus P_{(4)}, \mathbb{F}[\mathcal{O P}(3,1)] \cong P_{(3,1)} \oplus P_{(4)}$, and summing these gives

$$
\mathbb{F}\left[\mathcal{O P}_{4,2}\right] \cong P_{(1,3)} \oplus P_{(2,2)} \oplus P_{(3,1)} \oplus P_{(4)}^{\oplus 3} .
$$




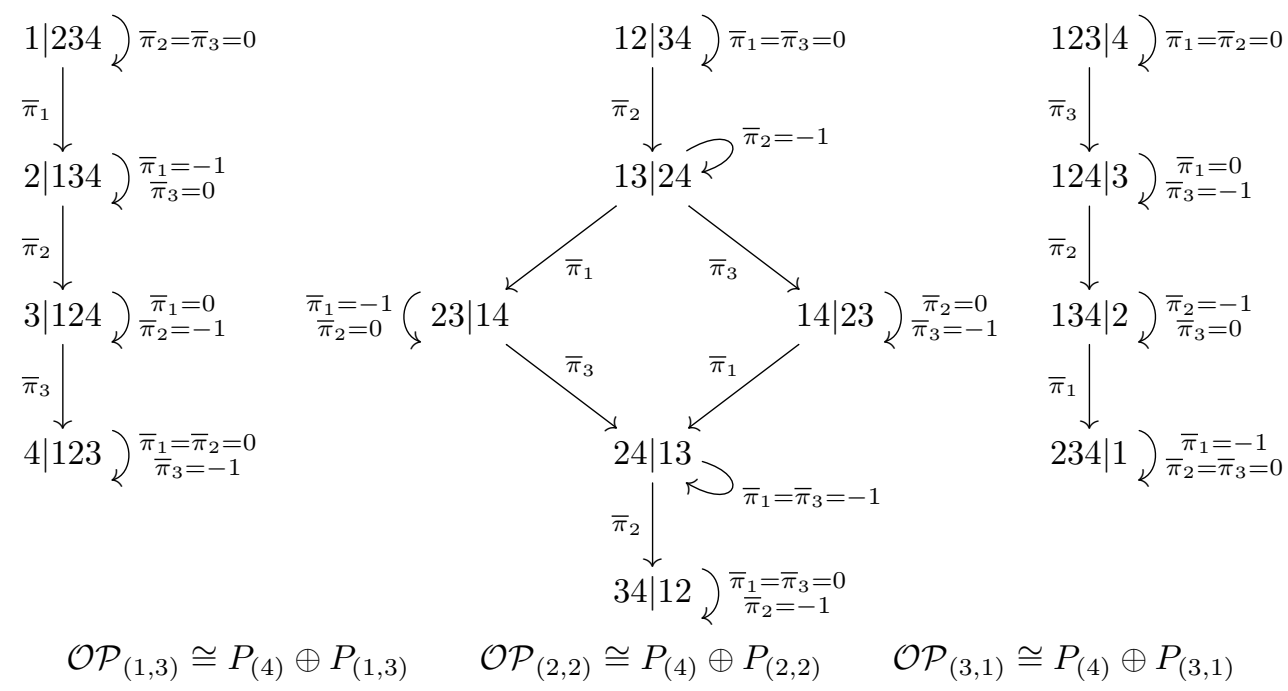

Figure 1. A decomposition of $\mathbb{F}\left[\mathcal{O P}_{4,2}\right]$

5.2. 0-Hecke ACTION ON POLynOmials. Our next task is to show that $S_{n, k}$ has the same isomorphism type as the $H_{n}(0)$-module of Proposition 5.3. To do this, we will need to study the action of $H_{n}(0)$ on the polynomial ring $\mathbb{F}\left[\mathbf{x}_{n}\right]$ via the isobaric Demazure operators $\pi_{i}$ defined in (7). Using the relation $\bar{\pi}_{i}=\pi_{i}-1$, we have

$$
\bar{\pi}_{i}(f):=\frac{x_{i+1} f-x_{i+1}\left(s_{i}(f)\right)}{x_{i}-x_{i+1}}, \quad \forall i \in[n-1], \forall f \in \mathbb{F}\left[\mathbf{x}_{n}\right] .
$$

Thus for an arbitrary monomial $x_{1}^{a_{1}} \cdots x_{n}^{a_{n}}$, we have

$$
\bar{\pi}_{i}\left(x_{1}^{a_{1}} \cdots x_{n}^{a_{n}}\right)= \begin{cases}\left(x_{1}^{a_{1}} \cdots x_{i-1}^{a_{i-1}} x_{i+2}^{a_{i+2}} \cdots x_{n}^{a_{n}}\right) \sum_{j=1}^{a_{i}-a_{i+1}} x_{i}^{a_{i}-j} x_{i+1}^{a_{i+1}+j} & a_{i}>a_{i+1} \\ 0 & a_{i}=a_{i+1} \\ -\left(x_{1}^{a_{1}} \cdots x_{i-1}^{a_{i-1}} x_{i+2}^{a_{i+2}} \cdots x_{n}^{a_{n}}\right) \sum_{j=0}^{a_{i+1}-a_{i}-1} x_{i}^{a_{i+1}+j} x_{i+1}^{a_{i}-j} & a_{i}<a_{i+1} .\end{cases}
$$

Using this we have the following triangularity result.

LEMMA 5.4. Let $\mathbf{d}=\left(d_{1} \geqslant \cdots \geqslant d_{n}\right)$ be a weakly decreasing vector of nonnegative integers and let $\mathbf{x}^{\mathbf{d}}=x_{1}^{d_{1}} \cdots x_{n}^{d_{n}}$ be the corresponding monomial in $\mathbb{F}\left[\mathbf{x}_{n}\right]$. Suppose $w \in \mathfrak{S}_{n}$ satisfies $\operatorname{Des}(w) \subseteq \operatorname{Des}(\mathbf{d})$. The polynomial $\bar{\pi}_{w}\left(\mathbf{x}^{\mathbf{d}}\right)$ has the form

$$
\bar{\pi}_{w}\left(\mathbf{x}^{\mathbf{d}}\right)=w\left(\mathbf{x}^{\mathbf{d}}\right)+\sum_{m \prec w\left(\mathbf{x}^{\mathbf{d}}\right)} c_{m} \cdot m
$$

for some $c_{m} \in \mathbb{F}$.

Proof. The proof is similar to [17, Lem. 4.1]. Observe that a monomial $m$ satisfies $m \prec \mathbf{x}^{\mathbf{d}}$ if and only if $m \prec w\left(\mathbf{x}^{\mathbf{d}}\right)$ for any permutation $w \in \mathfrak{S}_{n}$.

We induct on the length $\ell(w)$ of the permutation $w$. If $\ell(w)=0$, then $w$ is the identity permutation and the lemma is trivial. Otherwise, we may write $w=s_{j} v$, where $j \in[n-1]$ and $v \in \mathfrak{S}_{n}$ satisfies $\ell(w)=\ell(v)+1$. We have $j \in \operatorname{Des}\left(w^{-1}\right)$, 
$j \notin \operatorname{Des}\left(v^{-1}\right)$, and $\operatorname{Des}(v) \subseteq \operatorname{Des}(w) \subseteq \operatorname{Des}(\mathbf{d})$. By induction we have

$$
\bar{\pi}_{v}\left(\mathbf{x}^{\mathbf{d}}\right)=v\left(\mathbf{x}^{\mathbf{d}}\right)+\sum_{m \prec \mathbf{x}^{\mathbf{d}}} a_{m} \cdot m
$$

for some scalars $a_{m} \in \mathbb{F}$.

Since $j \notin \operatorname{Des}\left(v^{-1}\right)$, we have $v^{-1}(j)<v^{-1}(j+1)$ and thus $d_{v^{-1}(j)} \geqslant d_{v^{-1}(j+1)}$. Since $w v^{-1}(j)=s_{j}(j)>s_{j}(j+1)=w v^{-1}(j+1)$, there exists an element of $\left[v^{-1}(j), v^{-1}(j+\right.$ $1)-1]$ which belongs to $\operatorname{Des}(w) \subseteq \operatorname{Des}(\mathbf{d})$. This implies $d_{v^{-1}(j)}>d_{v^{-1}(j+1)}$. Then by (77), applying $\bar{\pi}_{j}$ to $v\left(\mathbf{x}^{\mathbf{d}}\right)=x_{v(1)}^{d_{1}} \cdots x_{v(n)}^{d_{n}}$ we have

$$
\bar{\pi}_{j}\left(v\left(\mathbf{x}^{\mathbf{d}}\right)\right)=s_{j} v\left(\mathbf{x}^{\mathbf{d}}\right)+\sum_{m^{\prime} \prec v\left(\mathbf{x}^{\mathbf{d}}\right)} b_{m^{\prime}} \cdot m^{\prime}=w\left(\mathbf{x}^{\mathbf{d}}\right)+\sum_{m^{\prime} \prec \mathbf{x}^{\mathbf{d}}} b_{m^{\prime}} \cdot m^{\prime}
$$

for some scalars $b_{m^{\prime}} \in \mathbb{F}$. On the other hand, (77) also implies that applying $\bar{\pi}_{j}$ to any monomial which is $\prec \mathbf{x}^{\mathbf{d}}$ will only yield terms which are also $\prec \mathbf{x}^{\mathbf{d}}$. Hence $\bar{\pi}_{w}\left(\mathbf{x}^{d}\right)$ has the desired form.

We will decompose the quotient $S_{n, k}$ into a direct sum of projective modules of the form $P_{\alpha, \beta}$ defined in (72). This decomposition will ultimately rest on the following lemma.

LEMmA 5.5. Let $\mathbf{d}=\left(d_{1} \geqslant \cdots \geqslant d_{n}\right)$ be a weakly decreasing sequence of nonnegative integers. Suppose $\alpha, \beta \models n$ such that $\alpha \preceq \beta$ and $\operatorname{Des}(\mathbf{d})=\operatorname{Des}(\beta)$. Then $H_{n}(0) \bar{\pi}_{w_{0}(\alpha)} \mathbf{x}^{\mathbf{d}}$ has basis

$$
\left\{\bar{\pi}_{w}\left(\mathbf{x}^{\mathbf{d}}\right): \operatorname{Des}(\alpha) \subseteq \operatorname{Des}(w) \subseteq \operatorname{Des}(\beta)\right\} .
$$

Furthermore, sending each element $\bar{\pi}_{w}\left(\mathbf{x}^{\mathbf{d}}\right)$ in the basis (81) to $\bar{\pi}_{w} \pi_{w_{0}\left(\beta^{c}\right)}$ gives an isomorphism $H_{n}(0) \bar{\pi}_{w_{0}(\alpha)} \mathbf{x}^{\mathbf{d}} \cong P_{\alpha, \beta}$ of $H_{n}(0)$-modules.

Proof. Let $1 \leqslant i \leqslant n-1$. If $i \notin \operatorname{Des}(\beta)$, then the monomial $\mathbf{x}^{\mathbf{d}}$ is symmetric in $x_{i}$ and $x_{i+1}$, so that $\bar{\pi}_{i}\left(\mathbf{x}^{\mathbf{d}}\right)=0$ by $(77)$. More generally, if $w \in \mathfrak{S}_{n}$ is such that $\operatorname{Des}(w) \nsubseteq \mathbb{D e s}(\beta)$ then $\bar{\pi}_{w}\left(\mathbf{x}^{\mathbf{d}}\right)=0$ because there exists a reduced expression for $w$ ending in $s_{i}$ for some $i \in \operatorname{Des}(w) \backslash \operatorname{Des}(\beta)$.

By the last paragraph and the fact that $w_{0}(\alpha)$ is the left weak Bruhat minimal permutation with descent set $\alpha$, the module $H_{n}(0) \bar{\pi}_{w_{0}(\alpha)} \mathbf{x}^{\mathbf{d}}$ is spanned by the set (81). This set is linearly independent and hence a basis for $H_{n}(0) \bar{\pi}_{w_{0}(\alpha)} \mathbf{x}^{\mathbf{d}}$, since by Lemma 5.4 and the equality $\operatorname{Des}(\mathbf{d})=\operatorname{Des}(\beta)$, any two distinct elements $\bar{\pi}_{w}\left(\mathbf{x}^{\mathbf{d}}\right)$ and $\bar{\pi}_{w^{\prime}}\left(\mathbf{x}^{\mathbf{d}}\right)$ of this set have neglex leading monomials $w\left(\mathbf{x}^{\mathbf{d}}\right)$ and $w^{\prime}\left(\mathbf{x}^{\mathbf{d}}\right)$, which are distinct by $\operatorname{Des}(w) \subseteq \operatorname{Des}(\mathbf{d})$ and $\operatorname{Des}\left(w^{\prime}\right) \subseteq \operatorname{Des}(\mathbf{d})$.

By Lemma 5.1, the module $P_{\alpha, \beta}$ has basis given by (73). Thus the assignment $\bar{\pi}_{w}\left(\mathbf{x}^{\mathbf{d}}\right) \mapsto \bar{\pi}_{w} \pi_{w_{0}\left(\beta^{c}\right)}$ induces a linear isomorphism from $H_{n}(0) \bar{\pi}_{w_{0}(\alpha)} \mathbf{x}^{\mathbf{d}}$ to $P_{\alpha, \beta}$. To check that this is an isomorphism of $H_{n}(0)$-modules, let $1 \leqslant i \leqslant n-1$. We compare the action of $\bar{\pi}_{i}$ on the bases (81) and (73) as follows. Let $w \in \mathfrak{S}_{n}$ satisfy $\operatorname{Des}(\alpha) \subseteq \operatorname{Des}(w) \subseteq \operatorname{Des}(\beta)$.

If $i \in \operatorname{Des}\left(w^{-1}\right)$, then there is a reduced expression for $w$ starting with $s_{i}$ and $\bar{\pi}_{i}$ acts by the scalar -1 on both $\bar{\pi}_{w}\left(\mathbf{x}^{\mathbf{d}}\right)$ and $\bar{\pi}_{w} \pi_{w_{0}\left(\beta^{c}\right)}$ since $\bar{\pi}_{i}^{2}=-\bar{\pi}_{i}$.

If $i \notin \operatorname{Des}\left(w^{-1}\right)$ and $\operatorname{Des}\left(s_{i} w\right) \subseteq \operatorname{Des}(\beta)$, then the polynomial $\bar{\pi}_{i} \bar{\pi}_{w}\left(\mathbf{x}^{\mathbf{d}}\right)=\bar{\pi}_{s_{i} w}\left(\mathbf{x}^{\mathbf{d}}\right)$ lies in the basis $(81)$ and the algebra element $\bar{\pi}_{i} \bar{\pi}_{w} \pi_{w_{0}\left(\beta^{c}\right)}=\bar{\pi}_{s_{i} w} \pi_{w_{0}\left(\beta^{c}\right)}$ lies in the basis (73).

If $i \notin \operatorname{Des}\left(w^{-1}\right)$ and $\operatorname{Des}\left(s_{i} w\right) \nsubseteq \subseteq \operatorname{Des}(\beta)$, we have $\bar{\pi}_{i} \bar{\pi}_{w}\left(\mathbf{x}^{\mathbf{d}}\right)=\bar{\pi}_{s_{i} w}\left(\mathbf{x}^{\mathbf{d}}\right)=0$ by the observation in the first paragraph. On the other hand, we also have $\bar{\pi}_{i} \bar{\pi}_{w} \pi_{w_{0}\left(\beta^{c}\right)}=$ $\bar{\pi}_{s_{i} w} \pi_{w_{0}\left(\beta^{c}\right)}=0$, since $s_{i} w$ has a reduced expression ending with $s_{j}$ for some $j \in$ $\operatorname{Des}\left(\beta^{c}\right)$ and $\bar{\pi}_{j} \pi_{w_{0}\left(\beta^{c}\right)}=0$ by the relation $\bar{\pi}_{j} \pi_{j}=0$. 
5.3. Decomposition of $S_{n, k}$. We begin by introducing a family of $H_{n}(0)$ submodules of $S_{n, k}$.

Definition 5.6. Let $A_{n, k}$ be the set of all pairs $(\alpha, \mathbf{i})$, where $\alpha \models n$ is a composition whose first part satisfies $\alpha_{1}>n-k$ and $\mathbf{i}=\left(i_{1}, \ldots, i_{n}\right)$ is a sequence of nonnegative integers satisfying

$$
k-\ell(\alpha) \geqslant i_{1} \geqslant \cdots \geqslant i_{n-k} \geqslant 0=i_{n-k+1}=\cdots=i_{n} .
$$

Given a pair $(\alpha, \mathbf{i}) \in A_{n, k}$, let $N_{\alpha, \mathbf{i}}$ be the $H_{n}(0)$-module generated by the image of the polynomial $\bar{\pi}_{w_{0}(\alpha)}\left(\mathbf{x}_{\alpha, \mathbf{i}}\right)$ in the quotient ring $S_{n, k}$.

For example, let $(n, k)=(6,3)$. Eliminating the $k=3$ trailing zeros from the $\mathbf{i}$ sequences, and omitting parentheses and commas from compositions $\alpha$ and sequences i, we have

$$
A_{6,3}=\left\{\begin{array}{c}
(411,000),(42,111),(42,110),(42,100),(42,000),(51,111), \\
(51,110),(51,100),(51,000),(6,222),(6,221),(6,220) \\
(6,211),(6,210),(6,200),(6,111),(6,110),(6,100),(6,000)
\end{array}\right\} .
$$

Recall that, if $\alpha \models n$ and if $\mathbf{i}$ is a length $n$ integer sequence, the composition $\alpha \cup \mathbf{i}=n$ is characterized by $\operatorname{Des}(\alpha \cup \mathbf{i})=\operatorname{Des}(\alpha) \cup \operatorname{Des}(\mathbf{i})$. When $(\alpha, \mathbf{i}) \in A_{n, k}$ we have the disjoint union decomposition $\operatorname{Des}(\alpha \cup \mathbf{i})=\operatorname{Des}(\alpha) \sqcup \operatorname{Des}(\mathbf{i})$. In fact, each element of $\operatorname{Des}(\mathbf{i})$ lies in the interval $1 \leqslant j \leqslant n-k$ whereas each element of $\operatorname{Des}(\alpha)$ lies in the interval $n-k+1 \leqslant j \leqslant n-1$.

It will turn out that the $N_{\alpha, \mathbf{i}}$ modules are special cases of the $P_{\alpha, \beta}$ modules. We will prove that if $(\alpha, \mathbf{i}) \in A_{n, k}$, then $N_{\alpha, \mathbf{i}} \cong P_{\alpha, \alpha \cup \mathbf{i}}$. To prove this fact, we will need a modification of the GS basis $\mathcal{G S}_{n, k}$ of $S_{n, k}$. This modified basis will come from the following lemma, which states that the collection of GS basis elements $\mathcal{G S}_{n, k}$ is related in a unitriangular way with the collection of polynomials

$$
\left\{\bar{\pi}_{w}\left(x_{\alpha, \mathbf{i}}\right):(\alpha, \mathbf{i}) \in A_{n, k}, \operatorname{Des}(\alpha) \subseteq \operatorname{Des}(w) \subseteq \operatorname{Des}(\alpha \cup \mathbf{i})\right\} .
$$

Lemma 5.7. Let $k \leqslant n$ be positive integers and endow monomials in $\mathbb{F}\left[\mathbf{x}_{n}\right]$ with the partial order $\prec$.

(i) Let $(\alpha, \mathbf{i}) \in A_{n, k}$ and $w \in \mathfrak{S}_{n}$ be such that $\operatorname{Des}(\alpha) \subseteq \operatorname{Des}(w) \subseteq \operatorname{Des}(\alpha \cup \mathbf{i})$. Then the unique $\prec$-leading term of $\bar{\pi}_{w}\left(\mathbf{x}_{\alpha, \mathbf{i}}\right)$ is $w\left(\mathbf{x}_{\alpha, \mathbf{i}}\right)=g s_{w, \mathbf{i}^{\prime}} \in \mathcal{G} \mathcal{S}_{n, k}$, where $\mathbf{i}^{\prime}=\left(i_{1}^{\prime}, \ldots, i_{n}^{\prime}\right)$ is related to $\mathbf{i}=\left(i_{1}, \ldots, i_{n}\right)$ by

$$
i_{j}^{\prime}=i_{j}-|\{r \in \operatorname{Des}(w) \cap[n-k]: r \geqslant j\}| .
$$

(ii) Let $g s_{w, \mathbf{i}^{\prime}} \in \mathcal{G S}_{n, k}$ be a GS basis element. Then $g s_{w, \mathbf{i}^{\prime}}$ is the unique $\prec$-leading term of $\bar{\pi}_{w}\left(\mathbf{x}_{\alpha, \mathbf{i}}\right)$ for some $w \in \mathfrak{S}_{n}$ and some $(\alpha, \mathbf{i}) \in A_{n, k}$ satisfying $\operatorname{Des}(\alpha) \subseteq$ $\operatorname{Des}(w) \subseteq \operatorname{Des}(\alpha \cup \mathbf{i})$ if and only if

- $\alpha \models n$ is characterized by $\operatorname{Des}(\alpha)=\operatorname{Des}(w) \backslash[n-k]$, and

- the sequence $\mathbf{i}=\left(i_{1}, \ldots, i_{n}\right)$ is related to the sequence $\mathbf{i}^{\prime}=\left(i_{1}^{\prime}, \ldots, i_{n}^{\prime}\right)$ by Equation (82).

Proof. (i) Since $\operatorname{Des}(w) \subseteq \operatorname{Des}(\alpha \cup \mathbf{i})$, Lemma 5.4 applies to show that the unique $\prec$-leading term of $\bar{\pi}_{w}\left(\mathbf{x}_{\alpha, \mathbf{i}}\right)$ is $w\left(\mathbf{x}_{\alpha, \mathbf{i}}\right)$. We need to show that

- the sequence $\mathbf{i}^{\prime}=\left(i_{1}^{\prime}, \ldots, i_{n}^{\prime}\right)$ is nonnegative, weakly decreasing, and satisfies $i_{1}^{\prime}<k-\operatorname{des}(w)$ and $i_{n-k+1}^{\prime}=\cdots=i_{n}^{\prime}=0$ so that the GS monomial $g s_{w, \mathbf{i}^{\prime}}$ makes sense and lies in $\mathcal{G} \mathcal{S}_{n, k}$, and

- we have $w\left(\mathbf{x}_{\alpha, \mathbf{i}}\right)=g s_{w, \mathbf{i}^{\prime}}$. 
It is clear that $i_{j}^{\prime}=i_{j}=0$ for $j>n-k$. We check that the sequence $\mathbf{i}^{\prime}$ is weakly decreasing. To see this, let $1 \leqslant j \leqslant n-k$ and note that

$$
i_{j}^{\prime}-i_{j+1}^{\prime}= \begin{cases}i_{j}-i_{j+1}-1 & j \in \operatorname{Des}(w) \cap[n-k], \\ i_{j}-i_{j+1} & j \notin \operatorname{Des}(w) \cap[n-k] .\end{cases}
$$

Since $\mathbf{i}$ is a weakly decreasing sequence and $i_{j}=i_{j+1} \operatorname{implies} j \notin \operatorname{Des}(\alpha \cup \mathbf{i}) \supseteq \operatorname{Des}(w)$, we conclude that $i_{j}^{\prime} \geqslant i_{j+1}^{\prime}$. Finally, we have $\operatorname{Des}(w) \cap[n-k]=\operatorname{Des}(w) \backslash \operatorname{Des}(\alpha)$ since the definition of $A_{n, k}$ implies $D(\alpha) \cap[n-k]=\varnothing$. Then

$$
i_{1}^{\prime}=i_{1}-|\operatorname{Des}(w) \cap[n-k]|=i_{1}-\operatorname{des}(w)+\ell(\alpha)-1<k-\operatorname{des}(w),
$$

so that $g s_{w, \mathbf{i}^{\prime}} \in \mathcal{G} \mathcal{S}_{n, k}$ is a genuine GS basis element.

Next, we show $w\left(\mathbf{x}_{\alpha, \mathbf{i}}\right)=g s_{w, \mathbf{i}^{\prime}}$. Let $1 \leqslant j \leqslant n$. Since $\operatorname{Des}(w) \cap[n-k]=\operatorname{Des}(w) \backslash$ $\operatorname{Des}(\alpha)$, it follows from $(82)$ that

$$
|\{r \in \operatorname{Des}(\alpha): r \geqslant j\}|+i_{j}=|\{r \in \operatorname{Des}(w): r \geqslant j\}|+i_{j}^{\prime} .
$$

This means that the variable $x_{w(j)}$ has the same exponent in $w\left(\mathbf{x}_{\alpha, \mathbf{i}}\right)$ as $g s_{w, \mathbf{i}^{\prime}}$. We conclude that $w\left(\mathbf{x}_{\alpha, \mathbf{i}}\right)=g s_{w, \mathbf{i}^{\prime}}$.

(ii) Let $g s_{w, \mathbf{i}^{\prime}} \in \mathcal{G} \mathcal{S}_{n, k}$. Suppose $g s_{w, \mathbf{i}^{\prime}}$ is the unique $\prec$-leading term of $\bar{\pi}_{w}\left(\mathbf{x}_{\alpha, \mathbf{i}}\right)$ for some $w \in \mathfrak{S}_{n}$ and some $(\alpha, \mathbf{i}) \in A_{n, k}$ satisfying $\operatorname{Des}(\alpha) \subseteq \operatorname{Des}(w) \subseteq \operatorname{Des}(\alpha \cup \mathbf{i})$.

The definition of $A_{n, k}$ implies $\operatorname{Des}(\alpha) \cap[n-k]=\varnothing$ and $\operatorname{Des}(\mathbf{i}) \subseteq[n-k]$. Thus $\operatorname{Des}(\alpha)=\operatorname{Des}(w) \backslash[n-k]$ and $\operatorname{Des}(w) \backslash \operatorname{Des}(\alpha)=\operatorname{Des}(w) \cap[n-k]$. Lemma 5.4 guarantees that $g s_{w, \mathbf{i}^{\prime}}=w\left(x_{\alpha, \mathbf{i}}\right)$. Comparing the power of the variable $x_{w(j)}$ on both sides of this equality gives $(82)$ for all $1 \leqslant j \leqslant n$.

Conversely, given $g s_{w, \mathbf{i}^{\prime}} \in \mathcal{G} \mathcal{S}_{n, k}$, define $\alpha$ and $\mathbf{i}$ as in the statement of the lemma. We have $(\alpha, \mathbf{i}) \in A_{n, k}$ and the unique $\prec$-leading term of $\bar{\pi}_{w}\left(\mathbf{x}_{\alpha, \mathbf{i}}\right)$ is $g s_{w, \mathbf{i}^{\prime}}$ by similar arguments to those above.

Lemma 5.7 can be used to derive a new basis for the quotient $S_{n, k}$. This basis will be helpful in decomposing $S_{n, k}$ into a direct sum of $H_{n}(0)$-modules of the form $N_{\alpha, \mathbf{i}}$.

LEMmA 5.8. Let $k \leqslant n$ be positive integers. The set of polynomials

$$
\left\{\bar{\pi}_{w}\left(\mathbf{x}_{\alpha, \mathbf{i}}\right):(\alpha, \mathbf{i}) \in A_{n, k}, w \in \mathfrak{S}_{n}, \operatorname{Des}(\alpha) \subseteq \operatorname{Des}(w) \subseteq \operatorname{Des}(\alpha \cup \mathbf{i})\right\}
$$

in $\mathbb{F}\left[\mathbf{x}_{n}\right]$ descends to a vector space basis of the quotient ring $S_{n, k}$. Moreover, for any $(\alpha, \mathbf{i}) \in A_{n, k}$ and any $w \in \mathfrak{S}_{n}$ with $\operatorname{Des}(\alpha) \subseteq \operatorname{Des}(w) \subseteq \operatorname{Des}(\alpha \cup \mathbf{i})$ we have

$$
\operatorname{deg}\left(\bar{\pi}_{w}\left(\mathbf{x}_{\alpha, \mathbf{i}}\right)\right)=\operatorname{deg}\left(\mathbf{x}_{\alpha, \mathbf{i}}\right)=\operatorname{maj}(\alpha)+|\mathbf{i}| .
$$

Proof. By Lemma 5.7, the polynomials in the statement satisfy the conditions of Lemma 4.2, and hence descend to a basis for $S_{n, k}$. The degree formula is clear.

In the coinvariant algebra case $k=n$, the basis of Lemma 5.8 appeared in [17]. As in [17], this modified GS-basis will facilitate analysis of the $H_{n}(0)$-structure of $S_{n, k}$.

THEOREM 5.9. Let $k \leqslant n$ be positive integers. For each $(\alpha, \mathbf{i}) \in A_{n, k}$, the set of polynomials

$$
\left\{\bar{\pi}_{w}\left(\mathbf{x}_{\alpha, \mathbf{i}}\right): w \in \mathfrak{S}_{n}, \operatorname{Des}(\alpha) \subseteq \operatorname{Des}(w) \subseteq \operatorname{Des}(\alpha \cup \mathbf{i})\right\}
$$

descends to a basis for $N_{\alpha, \mathbf{i}}$, and we have an isomorphism $N_{\alpha, \mathbf{i}} \cong P_{\alpha, \alpha \cup \mathbf{i}}$ of $H_{n}(0)$ modules by $\bar{\pi}_{w}\left(\mathbf{x}_{\alpha, \mathbf{i}}\right) \mapsto \bar{\pi}_{w} \pi_{w_{0}\left((\alpha \cup \mathbf{i})^{c}\right)}$. Moreover, the $H_{n}(0)$-module $S_{n, k}$ satisfies

$$
S_{n, k}=\bigoplus_{(\alpha, \mathbf{i}) \in A_{n, k}} N_{\alpha, \mathbf{i}} \cong \bigoplus_{\beta \models n} P_{\beta}^{\oplus\left(\begin{array}{l}
n-\ell(\beta) \\
k-\ell(\beta)
\end{array}\right)} \cong \mathbb{F}\left[\mathcal{O P} \mathcal{P}_{n, k}\right] .
$$


Proof. By Lemma 5.8, $S_{n, k}$ has a basis given by (86), which is the disjoint union of (88) for all $(\alpha, \mathbf{i}) \in A_{n, k}$. Combining this with Lemma 5.5, we have the basis (88) for $N_{\alpha, \mathbf{i}}$ and the desired isomorphism $N_{\alpha, \mathbf{i}} \cong P_{\alpha, \alpha \cup \mathbf{i}}$ for all $(\alpha, \mathbf{i}) \in A_{n, k}$. The decomposition $S_{n, k}=\bigoplus_{(\alpha, \mathbf{i}) \in A_{n, k}} N_{\alpha, \mathbf{i}}$ follows.

Next, let $\beta \models n$ and count the multiplicity of $P_{\beta}$ as a direct summand in $S_{n, k}$. Suppose $P_{\beta}$ is a direct summand of $N_{\alpha, \mathbf{i}}$ for some $(\alpha, \mathbf{i}) \in A_{n, k}$. Since $\operatorname{Des}(\alpha \cup \mathbf{i})$ is the disjoint union $\operatorname{Des}(\alpha) \sqcup \operatorname{Des}(\mathbf{i})$ and $\alpha \preceq \beta \preceq \alpha \cup \mathbf{i}$, we must have $\operatorname{Des}(\alpha)=$ $\operatorname{Des}(\beta) \backslash[n-k]$. It follows that the multiplicity of $P_{\beta}$ in $S_{n, k}$ equals the number of choices of $\mathbf{i}$ such that $(\alpha, \mathbf{i}) \in A_{n, k}$ and $\alpha \preceq \beta \preceq \alpha \cup \mathbf{i}$, where $\alpha$ is characterized by $\operatorname{Des}(\alpha)=\operatorname{Des}(\beta) \backslash[n-k]$.

We count the sequences $\mathbf{i}=\left(i_{1}, \ldots, i_{n}\right)$ of the above paragraph as follows. Since $\operatorname{Des}(\beta) \cap[n-k] \subseteq \operatorname{Des}(\mathbf{i})$, subtracting 1 from $i_{1}, \ldots, i_{r}$ for all $r \in \operatorname{Des}(\beta) \cap[n-k]$ gives a weakly decreasing sequence $\mathbf{i}^{\prime}=\left(i_{1}^{\prime}, \ldots, i_{n}^{\prime}\right)$ satisfying $i_{n-k+1}^{\prime}=\cdots=i_{n}^{\prime}=0$ and

$$
i_{1}^{\prime} \leqslant k-\ell(\alpha)-|\operatorname{Des}(\beta) \cap[n-k]|=k-\ell(\beta) .
$$

This gives a bijection from the collection of sequences $\mathbf{i}$ of the last paragraph and sequences $\mathbf{i}^{\prime}$ satisfying the conditions of the last sentence. The number of such sequences $\mathbf{i}^{\prime}$ is $\left(\begin{array}{c}n-\ell(\beta) \\ k-\ell(\beta)\end{array}\right)$, which equals the multiplicity of $P_{\beta}$ in $S_{n, k}$. Then Proposition 5.3 gives us $S_{n, k} \cong \mathbb{F}\left[\mathcal{O P} \mathcal{P}_{n, k}\right]$, as desired.

For example, let $(n, k)=(4,2)$. We have

$$
A_{4,2}=\{(31,0000),(4,1100),(4,1000)(4,0000)\} .
$$

We get the corresponding $N_{\alpha, \mathbf{i}}$ modules

$$
\begin{gathered}
N_{31,0000} \cong P_{(3,1),(3,1)} \cong P_{(3,1)} \quad N_{4,1100} \cong P_{(4),(2,2)} \cong P_{(4)} \oplus P_{(2,2)} \\
N_{4,1000} \cong P_{(4),(1,3)} \stackrel{\cong}{\cong} P_{(4)} \oplus P_{(1,3)} \quad N_{4,0000} \stackrel{\text { P }}{(4),(4)} \cong P_{(4)} .
\end{gathered}
$$

Combining this with Theorem 5.9, we have $S_{4,2} \cong P_{(2,2)} \oplus P_{(1,3)} \oplus P_{(3,1)} \oplus P_{(4)}^{\oplus 3} \cong$ $\mathbb{F}\left[\mathcal{O P}_{4,2}\right]$. The following picture illustrates this isomorphism via the action of $H_{4}(0)$ on the basis (86) of $S_{4,2}$ in Lemma 5.8. Note that the elements in this basis are polynomials in general, although they happen to be monomials in this example.

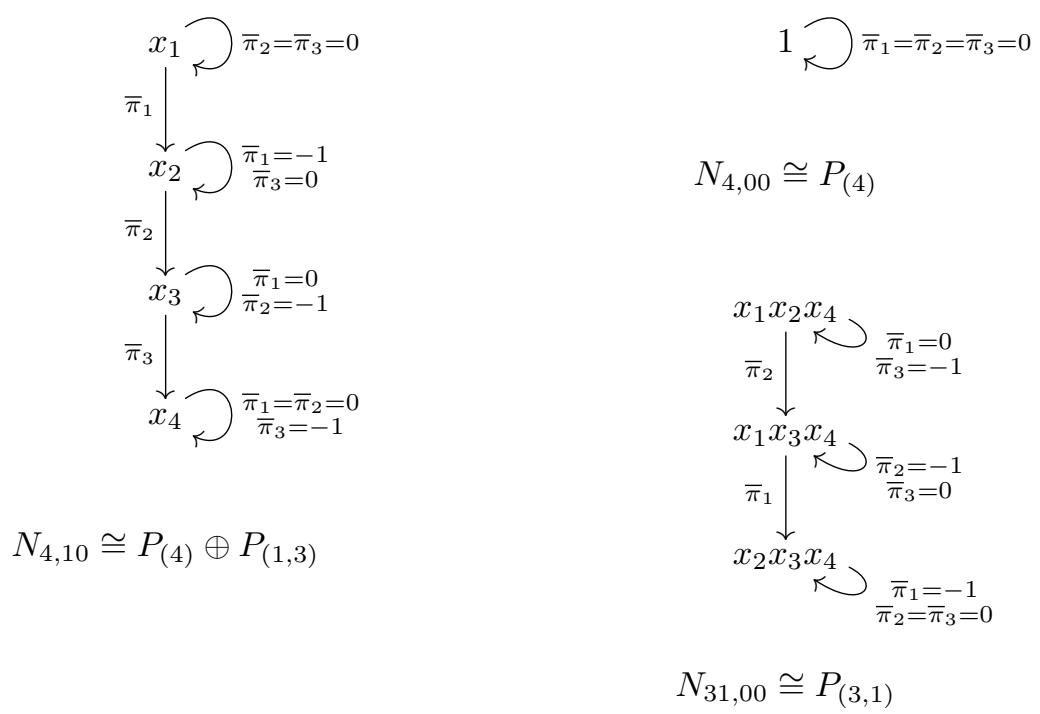




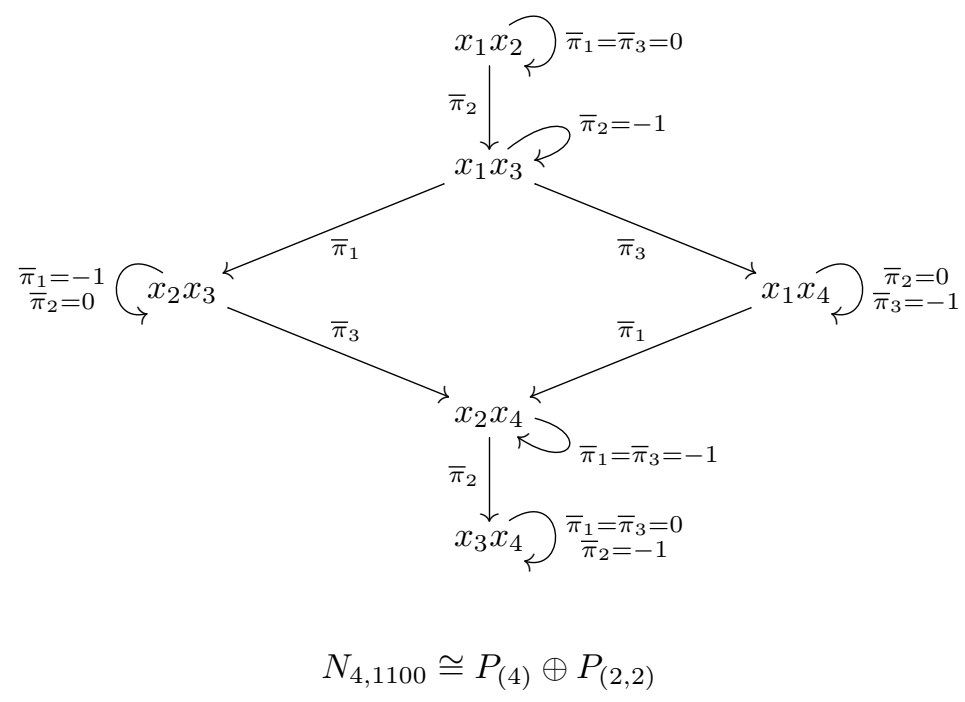

\section{Characteristic formulas}

In this section we derive formulas for the quasisymmetric and noncommutative symmetric characteristics of the modules $S_{n, k}$. To warm up, we calculate the degree-graded characteristics of the $N_{\alpha, \mathbf{i}}$ modules.

Recall that for $(\alpha, \mathbf{i}) \in A_{n, k}$ the module $N_{\alpha, \mathbf{i}}$ is the cyclic $H_{n}(0)$-module generated by the image of the polynomial $\bar{\pi}_{w_{0}(\alpha)}\left(\mathbf{x}_{\alpha, \mathbf{i}}\right)$ in the quotient $S_{n, k}$.

We adopt the length grading convention that the distinguished generator $\bar{\pi}_{w_{0}(\alpha)}\left(\mathbf{x}_{\alpha, \mathbf{i}}\right)$ of $N_{\alpha, \mathbf{i}}$ has length $\operatorname{inv}\left(w_{0}(\alpha)\right)$.

Lemma 6.1. Let $k \leqslant n$ be positive integers and let $(\alpha, \mathbf{i}) \in A_{n, k}$. The module $N_{\alpha, \mathbf{i}}$ is projective and the characteristics $\mathbf{c h}_{t}\left(N_{\alpha, \mathbf{i}}\right)$ and $\mathrm{Ch}_{q, t}\left(N_{\alpha, \mathbf{i}}\right)$ have the following expressions:

$$
\begin{aligned}
& \operatorname{ch}_{t}\left(N_{\alpha, \mathbf{i}}\right)=t^{\operatorname{maj}(\alpha)+|\mathbf{i}|} \sum_{\alpha \preceq \beta \preceq \alpha \cup \mathbf{i}} \mathbf{s}_{\beta}, \\
& \operatorname{Ch}_{q, t}\left(N_{\alpha, \mathbf{i}}\right)=t^{\operatorname{maj}(\alpha)+|\mathbf{i}|} \sum_{\substack{w \in \mathfrak{S}_{n} \\
\operatorname{Des}(\alpha) \subseteq \operatorname{Des}(w) \subseteq \operatorname{Des}(\alpha \cup \mathbf{i})}} q^{\operatorname{inv}(w)} F_{\mathrm{iDes}(w)},
\end{aligned}
$$

where in the second formula we view $N_{\alpha, \mathbf{i}}$ as a cyclic module generated by $\bar{\pi}_{w_{0}(\alpha)}\left(\mathbf{x}_{\alpha, \mathbf{i}}\right)$.

Proof. Theorem 5.9 and Lemma 5.1 show that $N_{\alpha, \mathbf{i}} \cong P_{\alpha, \alpha \cup \mathbf{i}} \cong \bigoplus_{\alpha \prec \gamma \prec \alpha \cup \mathbf{i}} P_{\gamma}$ is a direct sum of projective modules, so that $N_{\alpha, \mathbf{i}}$ is projective. As observed in the proof of Theorem 5.9, the set

$$
\left\{\bar{\pi}_{w}\left(\mathbf{x}_{\alpha, \mathbf{i}}\right): w \in \mathfrak{S}_{n}, \operatorname{Des}(\alpha) \subseteq \operatorname{Des}(w) \subseteq \operatorname{Des}(\alpha \cup \mathbf{i})\right\}
$$

is a basis for $N_{\alpha, \mathbf{i}}$. Since the degree of the polynomial $\bar{\pi}_{w}\left(\mathbf{x}_{\alpha, \mathbf{i}}\right)$ is $\operatorname{maj}(\alpha)+|\mathbf{i}|$, the formula for $\operatorname{ch}_{t}\left(N_{\alpha, \mathbf{i}}\right)$ follows from Theorem 5.9. For any $\ell \geqslant 0$, the term $N_{\alpha, \mathbf{i}}^{(\ell)}$ in the length filtration of $N_{\alpha, \mathbf{i}}$ has basis

$$
\left\{\bar{\pi}_{w}\left(\mathbf{x}_{\alpha, \mathbf{i}}\right): w \in \mathfrak{S}_{n}, \operatorname{Des}(\alpha) \subseteq \operatorname{Des}(w) \subseteq \operatorname{Des}(\alpha \cup \mathbf{i}), \ell(w) \geqslant \ell\right\} .
$$

The formula for $\mathrm{Ch}_{q, t}\left(N_{\alpha, \mathbf{i}}\right)$ follows. 
THEOREM 6.2. Let $k \leqslant n$ be positive integers. We have

$$
\begin{aligned}
\operatorname{ch}_{t}\left(S_{n, k}\right) & =\sum_{\alpha \models n} t^{\operatorname{maj}(\alpha)}\left[\begin{array}{l}
n-\ell(\alpha) \\
k-\ell(\alpha)
\end{array}\right]_{t} \mathbf{s}_{\alpha} \\
\mathrm{Ch}_{q, t}\left(S_{n, k}\right) & =\sum_{w \in \mathfrak{S}_{n}} q^{\operatorname{inv}(w)} t^{\operatorname{maj}(w)}\left[\begin{array}{l}
n-\operatorname{des}(w)-1 \\
k-\operatorname{des}(w)-1
\end{array}\right]_{t} F_{\mathrm{iDes}(w)} \\
& =\sum_{(w, \alpha) \in \mathcal{O P}_{n, k}} q^{\operatorname{inv}(w)} t^{\operatorname{maj}(w, \alpha)} F_{\mathrm{iDes}(w)}
\end{aligned}
$$

Proof. Theorem 5.9 gives a decomposition

$$
S_{n, k}=\bigoplus_{(\alpha, \mathbf{i}) \in A_{n, k}} N_{\alpha, \mathbf{i}}
$$

Combining this with Lemma 6.1 we have

$$
\begin{aligned}
& \operatorname{ch}_{t}\left(S_{n, k}\right)=\sum_{(\alpha, \mathbf{i}) \in A_{n, k}} t^{\operatorname{maj}(\alpha)+|\mathbf{i}|} \sum_{\alpha \preceq \beta \preceq \alpha \cup \mathbf{i}} \mathbf{s}_{\beta} \\
& =\sum_{\beta \models n} \sum_{\substack{\alpha, \mathbf{i}) \in A_{n, k} \\
\alpha \preceq \beta \preceq \alpha \cup \mathbf{i}}} t^{\operatorname{maj}(\alpha)+|\mathbf{i}|} \mathbf{s}_{\beta} .
\end{aligned}
$$

For each fixed composition $\beta \models n$, there exists $(\alpha, \mathbf{i}) \in A_{n, k}$ such that $\alpha \preceq \beta \preceq \beta \cup \mathbf{i}$ if and only if

- $\operatorname{Des}(\alpha)=\operatorname{Des}(\beta) \backslash[n-k]$ (so that $\alpha$ is uniquely determined by $\beta)$,

- the sequence $\mathbf{i}=\left(i_{1}, \ldots, i_{n}\right)$ satisfies $\operatorname{Des}(\mathbf{i})=\operatorname{Des}(\beta) \cap[n-k]$, and

- we have $k-\ell(\alpha) \geqslant i_{1} \geqslant \cdots \geqslant i_{n-k} \geqslant 0=i_{n-k+1}=\cdots=i_{n}$.

We obtain a sequence $\mathbf{i}^{\prime}=\left(i_{1}^{\prime}, \ldots, i_{n}^{\prime}\right)$ from $\mathbf{i}$ by subtracting 1 from $i_{1}, \ldots, i_{j}$ for all $j \in \operatorname{Des}(\mathbf{i})$. This gives a bijection between the sequences $\mathbf{i}$ satisfying the above requirements and the sequences $\mathbf{i}^{\prime}=\left(i_{1}^{\prime}, \ldots, i_{n}^{\prime}\right)$ such that

$$
k-\ell(\beta) \geqslant i_{1}^{\prime} \geqslant \cdots \geqslant i_{n-k}^{\prime} \geqslant 0=i_{n-k+1}=\cdots=i_{n}
$$

We also have

$$
\operatorname{maj}(\alpha)+|\mathbf{i}|=\operatorname{maj}(\beta)+|\mathbf{i}|-\operatorname{maj}(\mathbf{i})=\operatorname{maj}(\beta)+\left|\mathbf{i}^{\prime}\right| .
$$

It follows that

$$
\operatorname{ch}_{t}\left(S_{n, k}\right)=\sum_{\beta \models n} t^{\operatorname{maj}(\beta)}\left[\begin{array}{l}
n-\ell(\beta) \\
k-\ell(\beta)
\end{array}\right]_{t} \mathbf{s}_{\beta} .
$$

Lemma 6.1 and the decomposition (95) yield

$$
\begin{aligned}
\mathrm{Ch}_{q, t}\left(S_{n, k}\right) & =\sum_{(\alpha, \mathbf{i}) \in A_{n, k}} t^{\operatorname{maj}(\alpha)+|\mathbf{i}|} \sum_{\substack{w \in \mathfrak{S}_{n}: \\
\operatorname{Des}(\alpha) \subseteq \operatorname{Des}(w) \subseteq \operatorname{Des}(\alpha \cup \mathbf{i})}} q^{\operatorname{inv}(w)} F_{\mathrm{iDes}(w)} \\
& =\sum_{w \in \mathfrak{S}_{n}} q^{\operatorname{inv}(w)} \sum_{\substack{\operatorname{maj}(\alpha)+\mathbf{i}) \in A_{n, k}: \\
\operatorname{Des}(\alpha) \subseteq \operatorname{Des}(w) \subseteq \operatorname{Des}(\alpha \cup \mathbf{i})}} F_{\mathrm{iDes}(w)} \\
& =\sum_{w \in \mathfrak{S}_{n}} q^{\operatorname{inv}(w)} t^{\operatorname{maj}(w)}\left[\begin{array}{l}
n-\operatorname{des}(w)-1 \\
k-\operatorname{des}(w)-1
\end{array}\right]_{t} F_{\mathrm{iDes}(w)}
\end{aligned}
$$

where the last equality follows from the previous argument for $\mathbf{c h}_{t}\left(S_{n, k}\right)$ by setting $\operatorname{Des}(\beta)=\operatorname{Des}(w)$. 
Now recall that for an ordered set partition $(w, \alpha)=\left(B_{1}\left|B_{2}\right| \cdots \mid B_{k}\right) \in \mathcal{O} \mathcal{P}_{n, k}$ we have

$$
\operatorname{maj}(w, \alpha):=\operatorname{maj}(w)+\sum_{i: \max \left(B_{i}\right)<\min \left(B_{i+1}\right)}\left(\alpha_{1}+\cdots+\alpha_{i}-i\right) .
$$

For a fixed $w \in \mathfrak{S}_{n}$, there exists $\alpha \models n$ such that $(w, \alpha) \in \mathcal{O} \mathcal{P}_{n, k}$ if and only if $|\operatorname{Des}(w)|<k$ and $\operatorname{Des}(\alpha)$ contains all descents of $w$ together with $k-1-\operatorname{des}(w)$ many elements of $[n-1] \backslash \operatorname{Des}(w)$. Given a set of $k-1-\operatorname{des}(w)$ elements of $[n-1] \backslash \operatorname{Des}(w)$, we have $(w, \alpha)=\left(B_{1}|\cdots| B_{k}\right) \in \mathcal{O} \mathcal{P}_{n, k}$ determined in the above way, and this set corresponds to a lattice path from the lower-left corner to the upper-right corner of a $(k-1-\operatorname{des}(w)) \times(n-k)$ rectangle. The areas of the rows above this path are given by $\alpha_{1}+\cdots+\alpha_{i}-i$ for all $i \in[k-1]$ satisfying $\max \left(B_{i}\right)<\min \left(B_{i+1}\right)$. Thus

$$
\sum_{(w, \alpha) \in \mathcal{O P}_{n, k}} q^{\operatorname{inv}(w)} t^{\operatorname{maj}(w, \alpha)} F_{\mathrm{iDes}(w)}=\sum_{w \in \mathfrak{S}_{n}} q^{\operatorname{inv}(w)} t^{\operatorname{maj}(w)}\left[\begin{array}{l}
n-\operatorname{des}(w)-1 \\
k-\operatorname{des}(w)-1
\end{array}\right]_{t} F_{\mathrm{iDes}(w)} .
$$

This completes the proof.

REMARK 6.3. We can get the same characteristic $\mathrm{Ch}_{q, t}\left(S_{n, k}\right)$ as in Theorem 6.2 using a different decomposition of $S_{n, k}$ into cyclic modules coming from the $H_{n}(0)$-module isomorphisms

$$
S_{n, k} \cong \mathbb{F}\left[\mathcal{O P} \mathcal{P}_{n, k}\right] \cong \bigoplus_{\substack{\alpha=n \\ \ell(\alpha)=k}} \mathbb{F}\left[\mathcal{O P} \mathcal{P}_{\alpha}\right]
$$

provided by Theorem 5.9 and Proposition 5.3, without adjusting the length grading of each copy of the cyclic module $\mathbb{F}\left[\mathcal{O P} \mathcal{P}_{\alpha}\right]$ in $S_{n, k}$. The proof is somewhat messy and hence skipped.

The first expression for $\mathrm{Ch}_{q, t}\left(S_{n, k}\right)$ presented in Theorem 6.2 is related to an extension of the biMahonian distribution to ordered set partitions. More precisely, let $\sigma \in \mathcal{O P} \mathcal{P}_{n, k}$ be an ordered set partition and represent $\sigma$ as $(w, \alpha)$, where $w \in \mathfrak{S}_{n}$ is a permutation which satisfies $\operatorname{Des}(w) \subseteq \operatorname{Des}(\alpha)$. We define the length statistic $\ell(\sigma)$ by

$$
\ell(\sigma)=\ell(w, \alpha):=\operatorname{inv}(w)
$$

In the language of Coxeter groups, the permutation $w$ is the Bruhat minimal representative of the parabolic coset $w \mathfrak{S}_{\alpha}=w\left(\mathfrak{S}_{\alpha_{1}} \times \cdots \times \mathfrak{S}_{\alpha_{k}}\right)$, so that $\ell(\sigma)$ is the Coxeter length of this minimal element.

We have

$$
\sum_{\sigma \in \mathcal{O P}_{\alpha}} q^{\ell(\sigma)}=\left[\begin{array}{c}
n \\
\alpha_{1}, \ldots, \alpha_{k}
\end{array}\right]_{q}
$$

Summing Equation (97) over all $\alpha \models n$ with $\ell(\alpha)=k$ gives a different distribution than the generating function of maj:

$$
\sum_{\sigma \in \mathcal{O P}} q^{\operatorname{maj}(\sigma)}=\operatorname{rev}_{q}\left([k] !_{q} \cdot \operatorname{Stir}_{q}(n, k)\right),
$$

although these distributions both equal $[n] !_{q}$ in the case $k=n .^{(2)}$

By Theorem 6.2 we have

$$
\mathrm{Ch}_{q, t}\left(S_{n, k}\right)=\sum_{\sigma \in \mathcal{O} \mathcal{P}_{n, k}} q^{\ell(\sigma)} t^{\operatorname{maj}(\sigma)} F_{\mathrm{i} \operatorname{Des}(\sigma)},
$$

\footnotetext{
${ }^{(2)}$ There is a different extension of the inversion/length statistic on $\mathfrak{S}_{n}$ to $\mathcal{O} \mathcal{P}_{n, k}[22,27,23,15,16]$ whose distribution is $[k] !_{q} \cdot \operatorname{Stir}_{q}(n, k)$.
} 
where $F_{\mathrm{i} \operatorname{Des}(\sigma)}:=F_{\mathrm{iDes}(w)}$ for $\sigma=(w, \alpha)$. In other words, we have that $\mathrm{Ch}_{q, t}\left(S_{n, k}\right)$ is the generating function for the 'biMahonian pair' $\left(\ell\right.$, maj) on $\mathcal{O P}_{n, k}$ with quasisymmetric function weight $F_{\mathrm{iDes}(\sigma)}$.

We may also derive expressions for the degree-graded quasisymmetric characteristic $\mathrm{Ch}_{t}\left(S_{n, k}\right)$. It turns out that this quasisymmetric characteristic is actually a symmetric function since $S_{n, k}$ is projective and $\mathrm{Ch}\left(P_{\alpha}\right)=s_{\alpha} \in \mathrm{Sym}$ as given in (46). We give an explicit expansion of $\mathrm{Ch}_{t}\left(S_{n, k}\right)$ in the Schur basis.

COROLlary 6.4. Let $k \leqslant n$ be positive integers. We have

$$
\begin{aligned}
\mathrm{Ch}_{t}\left(S_{n, k}\right) & =\sum_{(w, \alpha) \in \mathcal{O} \mathcal{P}_{n, k}} t^{\operatorname{maj}(w, \alpha)} F_{\mathrm{iDes}(w)} \\
& =\sum_{w \in \mathfrak{S}_{n}} t^{\operatorname{maj}(w)}\left[\begin{array}{l}
n-\operatorname{des}(w)-1 \\
k-\operatorname{des}(w)-1
\end{array}\right]_{t} F_{\mathrm{iDes}(w)} \\
& =\sum_{\alpha \models n} t^{\operatorname{maj}(\alpha)}\left[\begin{array}{l}
n-\ell(\alpha) \\
k-\ell(\alpha)
\end{array}\right]_{t} s_{\alpha} .
\end{aligned}
$$

Moreover, the above symmetric function has expansion in the Schur basis given by

$$
\operatorname{Ch}_{t}\left(S_{n, k}\right)=\sum_{Q \in \operatorname{SYT}(n)} t^{\operatorname{maj}(Q)}\left[\begin{array}{l}
n-\operatorname{des}(Q)-1 \\
k-\operatorname{des}(Q)-1
\end{array}\right]_{t} s_{\text {shape }(Q)} .
$$

Proof. The first and second expressions for $\mathrm{Ch}_{t}\left(S_{n, k}\right)$ follow from Theorem 6.2 by setting $q=1$ in the expressions for $\mathrm{Ch}_{q, t}\left(S_{n, k}\right)$ given there. The third expression for $\mathrm{Ch}_{t}\left(S_{n, k}\right)$ follows from replacing $\mathbf{s}_{\alpha}$ by $s_{\alpha}$ in $\mathbf{c h}_{t}\left(S_{n, k}\right)$.

To derive Equation (103), we start with

$$
\mathrm{Ch}_{t}\left(S_{n, k}\right)=\sum_{w \in \mathfrak{S}_{n}} t^{\operatorname{maj}(w)}\left[\begin{array}{l}
n-\operatorname{des}(w)-1 \\
k-\operatorname{des}(w)-1
\end{array}\right]_{t} F_{\mathrm{iDes}(w)}
$$

and apply the Schensted correspondence. More precisely, the (row insertion) Schensted correspondence gives a bijection $w \mapsto(P(w), Q(w))$ from the symmetric group $\mathfrak{S}_{n}$ to ordered pairs of standard Young tableaux with $n$ boxes having the same shape. An example is given below.

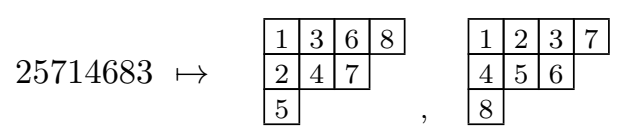

A descent of a standard tableau $P$ is a letter $i$ which appears in a row above the row containing $i+1$ in $P$. We let $\operatorname{Des}(P)$ denote the set of descents of $P$, and define the corresponding descent number $\operatorname{des}(P):=|\operatorname{Des}(P)|$ and major index $\operatorname{maj}(P):=\sum_{i \in \operatorname{Des}(P)} i$. Under the Schensted bijection we have $\operatorname{Des}(w)=\operatorname{Des}(Q(w))$, so that $\operatorname{des}(w)=\operatorname{des}(Q(w))$ and $\operatorname{maj}(w)=\operatorname{maj}(Q(w))$. Moreover, we have $w^{-1} \mapsto$ $(Q(w), P(w))$, so that $i \operatorname{Des}(w)=\operatorname{Des}(P(w))$.

Applying the Schensted correspondence, we see that

$$
\begin{aligned}
\mathrm{Ch}_{t}\left(S_{n, k}\right) & =\sum_{w \in \mathfrak{S}_{n}} t^{\operatorname{maj}(w)}\left[\begin{array}{l}
n-\operatorname{des}(w)-1 \\
k-\operatorname{des}(w)-1
\end{array}\right]_{t} F_{\mathrm{iDes}(w)} \\
& =\sum_{(P, Q)} t^{\operatorname{maj}(Q)}\left[\begin{array}{l}
n-\operatorname{des}(Q)-1 \\
k-\operatorname{des}(Q)-1
\end{array}\right]_{t} F_{\operatorname{Des}(P)},
\end{aligned}
$$


where the second sum is over all pairs $(P, Q)$ of standard Young tableaux with $n$ boxes satisfying shape $(P)=\operatorname{shape}(Q)$. Gessel [13] proved that for any $\lambda \vdash n$,

$$
\sum_{P \in \operatorname{SYT}(\lambda)} F_{\operatorname{Des}(P)}=s_{\lambda}
$$

where the sum is over all standard tableaux $P$ of shape $\lambda$. Applying Equation (106) gives

$$
\begin{aligned}
\sum_{(P, Q)} t^{\operatorname{maj}(Q)}\left[\begin{array}{l}
n-\operatorname{des}(Q)-1 \\
k-\operatorname{des}(Q)-1
\end{array}\right]_{t} & F_{\operatorname{Des}(P)}= \\
& =\sum_{Q} t^{\operatorname{maj}(Q)}\left[\begin{array}{l}
n-\operatorname{des}(Q)-1 \\
k-\operatorname{des}(Q)-1
\end{array}\right]_{t} \sum_{P \in \operatorname{SYT}(\operatorname{shape}(Q))} F_{\operatorname{Des}(P)} \\
& =\sum_{Q} t^{\operatorname{maj}(Q)}\left[\begin{array}{l}
n-\operatorname{des}(Q)-1 \\
k-\operatorname{des}(Q)-1
\end{array}\right]_{t} s_{\operatorname{shape}(Q)}
\end{aligned}
$$

as desired.

The Schur expansion of $\mathrm{Ch}_{t}\left(S_{n, k}\right)$ given in Corollary 6.4 coincides (after setting $q=t$ ) with the Schur expansion [16, Cor. 6.13] of the Frobenius image of the graded $\mathfrak{S}_{n}$-module $R_{n, k}$. That is, we have

$$
\mathrm{Ch}_{t}\left(S_{n, k}\right)=\operatorname{grFrob}\left(R_{n, k} ; t\right) .
$$

\section{Conclusion}

7.1. Macdonald polynomials and Delta conjecture. Equation (107) gives a connection between our work and the theory of Macdonald polynomials. More precisely, the Delta Conjecture of Haglund, Remmel, and Wilson [15] predicts that

$$
\Delta_{e_{k-1}}^{\prime} e_{n}=\operatorname{Rise}_{n, k-1}(\mathbf{x} ; q, t)=\operatorname{Val}_{n, k-1}(\mathbf{x} ; q, t),
$$

where $\Delta_{e_{k-1}}^{\prime}$ is the Macdonald eigenoperator defined by

$$
\Delta_{e_{k-1}}^{\prime}: \tilde{H}_{\mu} \mapsto e_{k-1}\left[B_{\mu}(q, t)-1\right] \cdot \tilde{H}_{\mu}
$$

and $\operatorname{Rise}_{n, k-1}(\mathbf{x} ; q, t)$ and $\operatorname{Val}_{n, k-1}(\mathbf{x} ; q, t)$ are certain combinatorially defined quasisymmetric functions; see [15] for definitions. By the work of Wilson [27] and Rhoades [23], we have the following consequence of the Delta Conjecture:

(110) $\operatorname{Rise}_{n, k-1}(\mathbf{x} ; q, 0)=\operatorname{Rise}_{n, k-1}(\mathbf{x} ; 0, q)=\operatorname{Val}_{n, k-1}(\mathbf{x} ; q, 0)=\operatorname{Val}_{n, k-1}(\mathbf{x} ; 0, q)$.

If we let $C_{n, k}(\mathbf{x} ; q)$ denote the common symmetric function in Equation (110), the work of Haglund, Rhoades, and Shimozono [16, Thm. 6.11] implies that

$$
\operatorname{grFrob}\left(R_{n, k} ; q\right)=\left(\operatorname{rev}_{q} \circ \omega\right) C_{n, k}(\mathbf{x} ; q),
$$

where $\omega$ is the standard involution on Sym sending $h_{d}$ to $e_{d}$ for all $d \geqslant 0$. Equation (107) implies that

$$
\mathrm{Ch}_{t}\left(S_{n, k}\right)=\left(\operatorname{rev}_{t} \circ \omega\right) C_{n, k}(\mathbf{x} ; t) .
$$

The derivation of $\operatorname{grFrob}\left(R_{n, k} ; q\right)$ in [16] has a different flavor from our derivation of $\mathrm{Ch}_{t}\left(S_{n, k}\right)$; the definition of the rings $R_{n, k}$ is extended to include a family $R_{n, k, s}$ involving a third parameter $s$. The $R_{n, k, s}$ rings are related to the image of the $R_{n, k}$ rings under a certain idempotent in the symmetric group algebra $\mathbb{Q}\left[\mathfrak{S}_{n}\right]$; this relationship forms the basis of an inductive derivation of $\operatorname{grFrob}\left(R_{n, k} ; q\right)$. The coincidence of $\mathrm{Ch}_{t}\left(S_{n, k}\right)$ and $\operatorname{grFrob}\left(R_{n, k} ; t\right)$ is mysterious to the authors. 
PROBLEM 7.1. Find a conceptual explanation of the identity

$$
\mathrm{Ch}_{t}\left(S_{n, k}\right)=\operatorname{grFrob}\left(R_{n, k} ; t\right) .
$$

7.2. TANisAKI IDEALS. Given a partition $\lambda \vdash n$, let $I_{\lambda} \subseteq \mathbb{F}\left[\mathbf{x}_{n}\right]$ denote the corresponding Tanisaki ideal (see [11] for a generating set of $I_{\lambda}$ ). When $\mathbb{F}=\mathbb{Q}$, the quotient $R_{\lambda}:=\mathbb{F}\left[\mathbf{x}_{n}\right] / I_{\lambda}$ is isomorphic to the cohomology ring of the Springer fiber attached to $\lambda$. The quotient $R_{\lambda}$ is a graded $\mathfrak{S}_{n}$-module. It is well known [11] that $\operatorname{grFrob}\left(R_{\lambda} ; q\right)=\operatorname{rev}_{q}\left(Q_{\lambda}^{\prime}(\mathbf{x} ; q)\right)$, where $Q_{\lambda}^{\prime}(\mathbf{x} ; q)$ is the dual Hall-Littlewood polynomial indexed by $\lambda$.

Huang proved that $I_{\lambda}$ is closed under the action of $H_{n}(0)$ on $\mathbb{F}\left[\mathbf{x}_{n}\right]$ if and only if $\lambda$ is a hook, so that the quotient $R_{\lambda}$ has the structure of a graded 0 -Hecke module for hook shapes $\lambda$ [17, Prop. 8.2]. Moreover, when $\lambda \vdash n$ is a hook, [17, Cor. 8.4] implies that $\mathrm{Ch}_{t}\left(R_{\lambda}\right)=\operatorname{grFrob}\left(R_{\lambda} ; t\right)=\operatorname{rev}_{t}\left(Q_{\lambda}^{\prime}(\mathbf{x} ; t)\right)$. When $\lambda \vdash n$ is not a hook, the quotient $R_{\lambda}$ does not inherit a 0 -Hecke action.

In this paper, we modified the ideal $I_{n, k}$ of [16] to obtain a new ideal $J_{n, k} \subseteq$ $\mathbb{F}\left[\mathbf{x}_{n}\right]$ which is stable under the action of $H_{n}(0)$ on $\mathbb{F}\left[\mathbf{x}_{n}\right]$. Moreover, we have $\mathrm{Ch}_{t}\left(\mathbb{F}\left[\mathbf{x}_{n}\right] / J_{n, k}\right)=\operatorname{grFrob}\left(\mathbb{Q}\left[\mathbf{x}_{n}\right] / I_{n, k} ; t\right)$. This suggests the following problem.

Problem 7.2. Let $\lambda \vdash n$. Define a homogeneous ideal $J_{\lambda} \subseteq \mathbb{F}\left[\mathbf{x}_{n}\right]$ which is stable under the 0-Hecke action on $\mathbb{F}\left[\mathbf{x}_{n}\right]$ such that

$$
\mathrm{Ch}_{t}\left(\mathbb{F}\left[\mathbf{x}_{n}\right] / J_{\lambda}\right)=\operatorname{grFrob}\left(R_{\lambda} ; t\right)=\operatorname{rev}_{t}\left(Q_{\lambda}^{\prime}(\mathbf{x} ; t)\right) .
$$

When $\lambda$ is a hook, the Tanisaki ideal $I_{\lambda}$ is a solution to Problem 7.2.

7.3. Generalization to Reflection groups. Let $W$ be a Weyl group. There is an action of the 0 -Hecke algebra $H_{W}(0)$ attached to $W$ on the Laurent ring of the weight lattice $Q$ of $W$. If $W$ has rank $r$, this Laurent ring is isomorphic to $\mathbb{F}\left[x_{1}, \ldots, x_{r}, x_{1}^{-1}, \ldots, x_{r}^{-1}\right]$. Huang described the 0 -Hecke structure of the corresponding coinvariant algebra [17, Thm. 5.3]. On the other hand, Chan and Rhoades [7] described a generalization of the ideal $I_{n, k}$ of [16] for the complex reflection groups $G(r, 1, n) \cong \mathbb{Z}_{r} \succ \mathfrak{S}_{n}$. It would be interesting to give an analog of the work in this paper for a wider class of reflection groups.

Acknowledgements. The authors are grateful to Jim Haglund, Adriano Garsia, Jeff Remmel, Mark Shimozono, and Andy Wilson for helpful conversations. The authors also thank two anonymous referees for their careful reading of this paper.

\section{REFERENCES}

[1] R. Adin, F. Brenti, and Y. Roichman, Descent representations and multivariate statistics, Trans. Amer. Math. Soc. 357 (2005), 3051-3082.

[2] E. Artin, Galois theory (second edition), Notre Dame Math Lectures, no 2., Notre Dame: University of Notre Dame, 1944.

[3] I. Assem, D. Simson, and A. Skowroński, Elements of the Representation Theory of Associative Algebras. Vol 1., London Mathematical Society Student Texts, Cambridge Univ. Press, Cambridge, 2006.

[4] C. Berg, N. Bergeron, F. Saliola, L. Serrano, and M. Zabrocki, Indecomposable modules for the dual immaculate basis of quasi-symmetric functions, Proc. Amer. Math. Soc. 143 (2015), 991-1000.

[5] F. Bergeron, Algebraic Combinatorics and Coinvariant Spaces, CMS Treatises in Mathematics. Taylor and Francis, Boca Raton, 2009.

[6] A. Björner and M. L. Wachs, Generalized quotients in Coxeter groups, Trans. Amer. Math. Soc. 308 (1988), 1-37.

[7] K.-T. J. Chan and B. Rhoades, Generalized coinvariant algebras for wreath products, Submitted, 2017. arXiv: 1701.06256.

[8] C. Chevalley, Invariants of finite groups generated by reflections, Amer. J. Math. 77 (1955), $778-782$. 
[9] D. Cox, J. Little, and D. O'Shea, Ideals, Varieties, and Algorithms (Third edition), Undergraduate Texts in Mathematics. Springer., New York, 1992.

[10] A. M. Garsia, Combinatorial methods in the theory of Cohen-Macaulay rings, Adv. Math. 38 (1980), 229-266.

[11] A. M. Garsia and C. Procesi, On certain graded $S_{n}$-modules and the $q$-Kostka polynomials, Adv. Math. 94 (1992), 82-138.

[12] A. M. Garsia and D. Stanton, Group actions on Stanley-Reisner rings and invariants of permutation groups, Adv. Math. 51 (1984), 107-201.

[13] I. Gessel, Multipartite P-partitions and inner products of skew Schur functions, Combinatorics and algebra. Contemp. Math., Vol. 34, Amer. Math. Soc., Providence, 1984, pp. 289-317.

[14] D. Grinberg and V. Reiner, Hopf algebras in combinatorics, arXiv:1509.8356.

[15] J. Haglund, J. Remmel, and A. T. Wilson, The Delta conjecture, Accepted, Trans. Amer. Math. Soc., 2016. arXiv: 1509.07058.

[16] J. Haglund, B. Rhoades, and M. Shimozono, Ordered set partitions, generalized coinvariant algebras, and the Delta conjecture, Submitted, 2016. arXiv:1509.07058.

[17] J. Huang, 0-Hecke actions on coinvariants and flags, J. Algebraic Combin. 40 (2014), 245-278.

[18] , A tableau approach to the representation theory of 0-Hecke algebras, Ann. Comb. 20 (2016), 831-868.

[19] D. Krob and J.-Y. Thibon, Noncommutative symmetric functions IV: Quantum linear groups and Hecke actions at $q=0$, J. Algebraic Combin. 6 (1997), 339-376.

[20] P. A. MacMahon, Combinatory Analysis (Volume 1), Cambridge University Press, Cambridge, 1915.

[21] P. N. Norton, 0-hecke algebras, J. Austral. Math. Soc. A 27 (1979), 337-357.

[22] J. Remmel and A. T. Wilson, An extension of MacMahon's Equidistribution Theorem to ordered set partitions, J. Combin. Theory Ser. A 134 (2015), 242-277.

[23] B. Rhoades, Ordered set partition statistics and the Delta Conjecture, J. Combin. Theory Ser. A 154 (2018), 172-217.

[24] G. C. Shephard and J. A. Todd, Finite unitary reflection groups, Can. J. Math. 6 (1954), 274-304.

[25] R. P. Stanley, Invariants of finite groups and their applications to combinatorics, Bull. Amer. Math. Soc. 1 (1979), 475-511.

[26] S. van Willigenburg and V. Tewari, Modules of the 0-Hecke algebra and quasisymmetric Schur functions, Adv. Math. 285 (2015), 1025-1065.

[27] A. T. Wilson, An extension of MacMahon's Equidistribution theorem to ordered multiset partitions, Electron. J. Combin. 23 (2016), P1.5.

JiA HuAng, University of Nebraska at Kearney, Department of Mathematics, Kearney, NE, 68849 (USA)

E-mail : huangj2@unk.edu

Brendon Rhoades, University of California, San Diego, Department of Mathematics, La Jolla, CA, 92093 (USA)

E-mail : bprhoades@math.ucsd.edu 